\title{
Functional localization and mechanisms of sequential effects in serial reaction time tasks
}

\author{
INES JENTZSCH \\ University of Glasgow, Glasgow, Scotland \\ and \\ WERNER SOMMER \\ Humboldt University, Berlin, Germany
}

\begin{abstract}
Reaction times (RTs) to randomly ordered stimuli are influenced in various ways by the sequence of preceding events. Depending on the response-stimulus interval and stimulus-response compatibility, cost-only or cost-benefit patterns can be observed. In order to localize these effects within the informationprocessing system, different sequential patterns were induced in overt performance. RTs and amplitude developments of the lateralized readiness potential (LRP) across several trials indicated the accumulation of residual traces as a possible mechanism underlying sequential effects. Analysis of LRP onsets indicated two possible loci of action of such traces. Whereas in motoric stages trace accumulation appeared to produce processing advantages only for continued event repetitions, without corresponding costs for discontinuations, cost-benefit patterns were consistently observed in premotoric stages.
\end{abstract}

In randomly ordered stimulus presentations, such as those used in many experimental situations, choice reaction times (RTs) are strongly affected by the sequence of preceding stimuli and responses. These sequential effects take different shapes, depending on a number of experimental factors. The present article addresses the mechanisms underlying these effects and the locus of their actions within the information-processing system.

In describing sequential effects, it is convenient to classify each event as a repetition (R) or an alternation (A) of the preceding event. Because the consequences of the immediately preceding event and of those events earlier in the sequence for the processing of a current stimulus can be quite different, they are often distinguished as firstorder (FO) and higher order ( $\mathrm{HO})$ effects, respectively. The type of $\mathrm{FO}$ and $\mathrm{HO}$ effects obtained in a given experimental situation depends on a number of factors (e.g., Bertelson \& Renkin, 1966; Hale, 1967; Kirby, 1976; Soetens, Boer, \& Hueting, 1985; Vervaeck \& Boer, 1980)—for example, on the response stimulus interval (RSI) and stimulus-response compatibility. In the following description, we will refer only to two-choice RT tasks, the situation encountered most commonly.

When conventional long RSIs ( $>500 \mathrm{msec}$ in compatible stimulus-to-response mappings) are used, the effects of the HO sequence greatly depend on whether the current event is a repetition or an alternation. For example,

We thank Hartmut Leuthold and Eric Soetens for helpful discussions and comments on preliminary versions of this article. Correspondence concerning this article should be addressed to I. Jentzsch, University of Glasgow, Department of Psychology, 58 Hillhead Street, Glasgow G12 8QB, Scotland (e-mail: i.jentzsch @ psy.gla.ac.uk). if a run of stimulus repetitions is continued by the present stimulus (RRRR, time scale running from left to right, the current event being italicized), subjects respond much faster than when the repetition run is terminated by an alternation (RRRA). Likewise, continued runs of alternations are responded to faster than discontinued runs (AAA $A$ vs. AAAR). Such a pattern, in which the effect of a given $\mathrm{HO}$ sequence is inverted when the FO event changes from $\mathrm{R}$ to $\mathrm{A}$, is termed a cost-benefit pattern (e.g., Kirby, 1976; Soetens et al., 1985; Vervaeck \& Boer, 1980; for an illustration of this pattern, see Figure 1).

In sharp contrast, the HO pattern observed in short-RSI conditions does not depend on whether the current event is a repetition or an alternation. Here, RTs after runs of repetitions are always faster than those after runs of alternations, independently of whether the current event is a repetition or an alternation (see Figure 5 for an illustration). That is, depending on the point of view, runs of repetitions are always beneficial, or conversely, runs of alternations are always costly. Therefore, the typical HO pattern at short RSIs is called a benefit-only or a cost-only pattern ${ }^{1}$ (Kirby, 1976; Vervaeck \& Boer, 1980). Importantly, in less compatible stimulus-to-response mappings, cost-only patterns are observed also at longer RSIs (Soetens et al., 1985).

Conditions that produce HO cost-only patterns often also yield a global RT advantage for FO repetitions over alternations, which is termed an $F O$ repetition effect. In contrast, HO cost-benefit patterns are often accompanied by FO alternation effects - that is, shorter RTs to an FO alternation than to a repetition (e.g., Kirby, 1972, 1976; Soetens et al., 1985). However, these associations are not universal. For example, despite clear HO costbenefit patterns, FO alternation effects were absent in the 


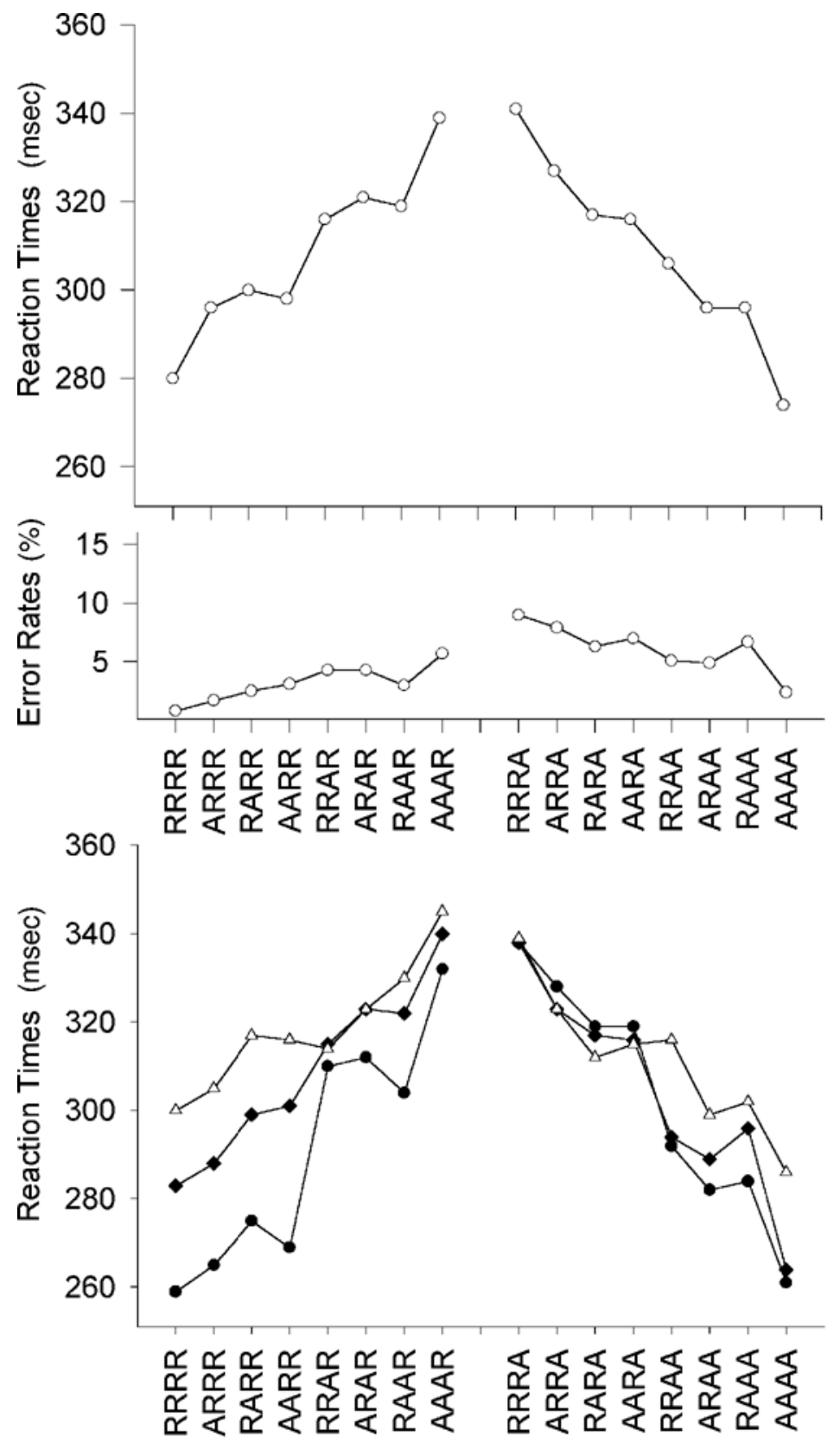

$\longrightarrow$ Bin 1 (fast) $\longrightarrow$ Bin 3 (medium) $\longrightarrow$ Bin 5 (slow)

Figure 1. Experiment 1: sequential effects in mean reaction times (RTs) (top), in mean error rates (middle), and in RTs partitioned according to the speed of the preceding response (bottom; mean RTs of the fastest, the slowest, and the middle of five equal bins). The variables are plotted as a function of first-order repetitions and alternations of the preceding stimulus ( $R$ and $A$, left and right sides of each panel, respectively) and the eight higher order sequences within each first-order sequence, ordered from three repetitions (RRR) to three alternations (AAA).

spatially compatible tasks of Remington (1969) and Sommer, Leuthold, and Soetens (1999) and in the noncompatible or symbolic mappings of Ford, DuncanJohnson, Pfefferbaum, and Kopell (1982) and Duncan-
Johnson, Roth, and Kopell (1984). Likewise, the costonly pattern found in short-RSI conditions is not always accompanied by an FO repetition effect (Melis, Soetens, $\&$ van der Molen, 2002; Sommer et al., 1999). 
In the past, different suggestions have been made for the origin of cost-benefit patterns (e.g., Laming, 1969) and cost-only patterns (e.g., Soetens, Deboeck, \& Hueting, 1984). Interestingly, most models assume that a major source for sequential patterns are residual activation traces left by previous stimulus-response cycles. Trace conceptions assume that the presence of traces facilitates processing if the current stimulus requires a similar kind of processing. In contrast, if processing of the present stimulus differs from the trace, there is either no advantage or even a disadvantage.

Trace accounts have been seen as contributing to subjective expectancy, a concept commonly used for explaining cost-benefit patterns (e.g., Kirby, 1980). If subjects expect a specific event, expectancy conforming will have processing advantages, whereas processing of nonconforming events will suffer costs. In his model, Laming (1969) suggested that three mechanisms contribute to subjective expectancy: residual traces left from previous processing cycles, an expectation for a further alternation after a run of alternations, and the global stimulus and alternation probability.

Interestingly, contributions of residual activation traces have also been included in a model explaining cost-only patterns in short-RSI situations (Soetens et al., 1984). However, a clearer inspection of this model shows that the trace mechanism actually produces a cost-benefit pattern, together with an FO repetition effect. Thus, the cost-only pattern, with its independence of the actual transition, must be produced by additional factors-for example, the sharpening of an internal standard for identifying the incoming signals (Laming, 1968) or an inductionlike inhibition of the alternative processing channel (Vervaeck \& Boer, 1980), both being included in the model of Soetens et al. (1984). Moreover, other possible mechanisms might also relate to monitoring of the executed response (Kirby, 1980; Welford, 1967, 1976) and to preparation for upcoming stimulus processing (Rabbitt, 1980). In the present article, we will focus on the trace account for sequence-dependent costs and benefits in two-choice RT tasks; therefore, we will not attempt to distinguish between the alternative accounts of cost-only patterns.

The contributions of memory traces to HO cost-benefit patterns might vary not only as a function of RSI, but also as a function of other factors, such as stimulus-response compatibility. Usually, it is assumed that traces decay as a function of time and that any new traces are added to the existing ones. The conception that traces decay over time implies that any sequential effects based on these traces should decrease as a function of increasing intervals between trials. This is in line with observed influences of increasing intertrial intervals (ITIs) on FO repetition effects (Entus \& Bindra, 1970; Hale, 1967; Kirby, 1976; Soetens et al., 1985; but see Rabbitt, 1980). Evidence for automatic, time-decaying activation also comes from studies investigating response code activation in the Simon task (e.g., De Jong, Liang, \& Lauber, 1994; Hommel, 1994). On the other hand, Ells and Gotts (1977) and Pash- ler and Baylis (1991) found the strength of the repetition effect to be determined not by the interval between successive trials, but only by the occurrence of intervening events itself. This poses a problem for the idea that traces passively depend merely on the passage of time.

In the present study, we sought evidence from both behavioral and electrophysiological observations for the involvement of passive, time-dependent trace accumulation and decay in the emergence of sequential patterns. One prediction that can be derived from this idea is that within a given experimental condition, one should expect a larger influence of passive activation traces if the response preceding the current stimulus were relatively fast. When stimulus presentation depends on the interval following the response (RSI), as is the case in the present experiments, the ITI is a direct function of the preceding RT. For fast preceding reactions, the interval between successive trials is reduced, and therefore, the influence of traces should be stronger if trace decay depends on the time between successive trials (passive trace hypothesis). In this case, one should find smaller cost-benefit and smaller FO repetition effects after long than after short preceding RTs. If, on the other hand, only trial occurrence itself determines trace activation, no difference between slow and fast preceding reactions should be observed.

Post hoc partitioning of RTs into those following slow and those following fast trials can only yield correlational evidence. Therefore, a different view on residual traces was sought by considering the amplitude of the lateralized readiness potential (LRP) across several consecutive trials. In recent years, the LRP has become a well-established tool for studying chronometrical questions in cognitive psychology (Coles, 1989; De Jong, Wierda, Mulder, \& Mulder, 1988). The LRP is based on a negative-going shift, the readiness potential, in the electroencephalogram (EEG) that precedes voluntary movements. The readiness potential exhibits greater negativity over motor areas contralateral to the responding hand as soon as the response hand is selected. The LRP extracts this response-related asymmetry by subtracting the event-related activity at the ipsilateral electrode site $\left(\mathrm{C}^{\prime}\right.$ ' for left-hand responses and $\mathrm{C}^{\prime}$ ' for right-hand responses) from the activity measured on the contralateral electrode site. A correct response activation is expressed in a negative-going LRP, whereas a positive LRP reflects activation of the incorrect response hand.

The idea of using the LRP for investigating the trace hypothesis was initiated by unpublished observations that LRP amplitude elicited by a given stimulus relative to a prestimulus baseline decreases with the run of preceding repetitions, particularly in short-RSI conditions. That means that runs of repetitions yield smaller LRP amplitudes than do alternations. A possible reason for such an amplitude reduction might be a summation of LRP activation across several trials, leading to amplitude saturation, captured by the baseline, and thus reducing the additional activation elicited by the present stimulus. Considering the 
development of the LRP across several successive trials might indicate trace accumulation in response-related processes. Traces and, hence, LRP amplitude across several trials should increase in strength with the length of the preceding repetition run and decrease with increasing intervals between repetitions - that is, with RSI.

A second question addressed in the present paper concerns the locus of sequential effects within the informationprocessing system. Although there have been numerous previous attempts to do so, they have focused on FO effects that may not allow a generalization to $\mathrm{HO}$ effects. The most frequently used approach to localization is the additive-factors method (AFM; Sternberg, 1969), which states that interactions between two experimental factors in RT indicate that these factors influence at least one processing stage in common. Thus, if sequential effects interact with a second experimental factor, they should involve a processing stage that is also affected by the other factor.

With the AFM method, it has been found that FO repetition effects are diminished with increasing stimulusresponse compatibility (Bertelson, 1963; Kornblum, 1969). Because compatibility is held to affect the duration of the response selection stage, this stage appears to be at least one of the loci where FO repetition benefits act. In a similar way, the increase of the FO repetition effect with increasing number of alternative choices (Biederman \& Stacy, 1974; Kornblum, 1975) can be interpreted as indicating a locus at response selection. Although not formally tested, $\mathrm{HO}$ patterns also appear to interact with stimulus-response compatibility (Soetens et al., 1985), pointing in the same direction.

A method that broadly distinguishes between perceptual and response-related loci of effects is the information reduction procedure (IRP). Here, several stimuli are mapped onto a smaller set of several responses that allows distinguishing between three types of trials. In socalled identical trials both the stimulus and the response are repeated, in equivalent trials only the response is repeated, and in different trials both the stimulus and the response alternate relative to the preceding trial. If RTs for identical trials are faster than those for both equivalent and different trials, the effect is considered to be stimulus related. If RTs are faster for equivalent than for different trials, the repetition effect is said to be response related. Note that according to this method, the responserelated stages cover both response execution and response selection.

Studies in which the IRP method has been used have mainly examined the locus of the FO repetition effect, yielding somewhat inconsistent results (for a review, see Pashler \& Baylis, 1991). With categorizable two-to-one mappings of numbers to response keys, Bertelson (1965) and Rabbitt (1968, practiced subjects) found approximately equal RTs in identical and equivalent trials but substantially slower responses in different trials, suggesting a response-related locus of the repetition effect.
For unpracticed subjects in Rabbitt's (1968) study, as well as for noncategorizable two-to-one mappings (Smith, 1968), identical trials were faster than both equivalent and different trials, indicative of stimulus relatedness. Accordingly, repetition benefits seem to be produced in response-related stages in the case of categorizable mappings and in stimulus-related stages for noncategorizable mappings and unpracticed subjects. Pashler and Baylis concluded from their findings that the repetition effect in tasks with easily classifiable stimuli is caused mainly by the creation and strengthening of links that shortcut the response selection stage. This is in line with Welford's (1976) suggestion that the repetition speed-up originates in the stage of mentally "translating" between stimulus and response (response selection).

Hitherto, only one study has investigated the locus of HO sequential dependencies by applying the IRP (Soetens, 1998). This study used a two-to-one mapping with the stimulus dimensions left-right and up-down. The results suggest a response-related locus of the cost-benefit pattern, but only if the spatial stimulus-response arrangement is compatible. In the case of an incompatible arrangement (Simon task) the response-related effect in the relevant dimension disappears, but now a cost-benefit pattern is found in the irrelevant (compatible) stimulus dimension. Cost-only patterns were found to be mainly stimulus specific, although response-related effects also were found for some stimulus-response arrangements.

The complexity of the results with the IRP may relate to the complex stimulus-response mapping involved. Especially problematic may be the strongly reduced a priori probability of identical stimulus repetitions and the increased probability of stimulus alternations in such tasks. As explicated in the model of Laming (1969), subjective expectancy is strongly influenced both by the a priori probability of stimuli and by alternations. Therefore, independent evidence for the applicability of the IRP findings to the more common two-choice tasks appears to be necessary. The aim of the present study was to directly examine the locus of $\mathrm{HO}$ sequential effects in two-choice tasks by recording the LRP.

The LRP is considered to index selective response activation (e.g., Coles, 1989; De Jong et al., 1988). It is assumed that the LRP begins to deviate from baseline as soon as information about the response hand is available. Hence, the locus of experimental effects can be inferred by analyzing LRP onsets obtained in waveforms timelocked either to response signal onset or to the overt response (Leuthold, Sommer, \& Ulrich, 1996; Osman \& Moore, 1993). The interval from response signal onset to stimulus-locked LRP onset (S-LRP interval) indicates the duration of those processes occurring before the start of the LRP. The interval between response-locked LRP onset and the overt response (LRP-R interval) indicates the duration of those processes that occur after LRP onset. That is, the S-LRP and the LRP-R intervals can be used as chronometrical markers for premotoric and motoric 
processing, respectively. These markers were used in the present study in order to localize the various sequential patterns within the information-processing system.

In sum, the aim of present study was to investigate the locus of sequential effects within information processing by assessing electrophysiological markers for stimulusand response-related processing duration. By considering sequential effects as a function of the preceding RT and analyzing long-term amplitude development of the LRP, direct evidence for the contributions of activation traces to cost-benefit and FO repetition effects could be sought.

In all, three experiments were conducted, for which different sequential patterns were expected. The first two experiments were spatially compatible with long- and short-RSI conditions, respectively. In the long-RSI condition (Experiment 1), a cost-benefit pattern in performance was expected, whereas the short-RSI condition (Experiment 2) was expected to yield a cost-only pattern. Experiment 3 used the same long-RSI condition as that in Experiment 1 but a less compatible stimulus-to-response mapping (color to location). Because decreasing compatibility at a given RSI shifts cost-benefit patterns toward cost-only, a mixture of both was expected in Experiment 3.

\section{EXPERIMENT 1}

\section{Method}

Subjects. Ten subjects ( 8 males) were tested who were between 18 and 37 years of age (mean, 26.8 years) and strongly righthanded, with laterality quotients greater than +60 (Oldf ield, 1971).

Stimuli, Apparatus, and Procedure. The stimulus was a white dot ( $5 \mathrm{~mm}$ in diameter) presented, in random order and equiprobably, $10 \mathrm{~mm}$ above or below a fixation line (4 $\mathrm{mm}$ in length) on a monitor. Viewing distance was $1 \mathrm{~m}$. The duration of the stimulus presentation was $60 \mathrm{msec}$, and an RSI of $700 \mathrm{msec}$ was applied. Two buttons mounted $15 \mathrm{~cm}$ apart on a vertical response panel in front of the subject were used for recording responses. The index fingers of the left and right hands operated the two keys. The assignment of the fingers to the keys was counterbalanced across subjects. The subjects were instructed to press the top and bottom keys in response to the dot above and below the fixation line, respectively. The subjects were instructed to make fast and accurate choice responses to the two stimuli. In one session, a total of 3,960 stimuli were presented. After each block of 330 trials, there was a short rest. The subjects were advised to avoid eye movements or blinks during the experiment.

Electrophysiological recordings. The EEG from 32 different midline and lateral sites were recorded with Sn-electrodes and Beckman Electrolyte paste. Here, we will only report results from the lateral electrode sites near the hand area of the primary motor cortex (C3', C4', C3, and C4). Analyses of different aspects of the data, which were concerned with topographical analysis and source localization of the P300 component, using signals from all 32 electrode sites, have been reported elsewhere (Jentzsch \& Sommer, 2001). All signals were amplified with a bandpass of $0-40 \mathrm{~Hz}(-3-\mathrm{dB}$ attenuation, 12-dB rolloff/octave) and were digitized at a rate of $200 \mathrm{samples} / \mathrm{sec}$. The electrophysiological signals were continuously recorded together with triggers for stimulus and response events.

Off line, the continuous EEG record was separated into four types of epochs. In order to analyze short-term peri-event activity, epochs of $1 \mathrm{sec}$ were used. Stimulus-synchronized epochs contained $200 \mathrm{msec}$ of prestimulus activity, and response-synchronized epochs contained $600 \mathrm{msec}$ of preresponse activity. In order to analyze long-term developments of LRP activity across several stimuli, epochs of $6.5 \mathrm{sec}$ were obtained with $5.5 \mathrm{sec}$ of prestimulus activity. The averaged ERPs were digitally low-pass filtered at $10 \mathrm{~Hz}$ $(-3 \mathrm{~dB})$.

Data analysis. For data analysis, only trials with correct keypresses and RTs of between 100 and 1,000 msec and without EEG and electroocular artifacts $(<80 \mu \mathrm{V})$ were considered. Each event was coded according to 16 fourth-order stimulus sequences, disregarding the physical event and considering only whether it constituted a repetition or an alternation with respect to its predecessor. For the analysis of sequential effects, we consistently used the scheme of Soetens et al. (1985). The 16 sequences were subdivided into those for which the actual stimulus was a repetition and those for which it was an alternation of the second-to-last stimulus (FO sequences). For each FO sequence, $8 \mathrm{HO}$ sequences were ordered as follows: RRR, ARR, RAR, AAR, RRA, ARA, RAA, and AAA.

All dependent variables were subjected to repeated measures analyses of variance (ANOVAs), with factors FO stimulus sequence (levels R and A) and HO sequence (levels RRR to AAA). If any main effect or interaction was significant, the linear trends across the levels of this variable were also considered. ${ }^{2}$ Conservative (Huynh-Feld) $F$ tests were used throughout.

As criteria for the presence and type of HO sequential effects, we used the slopes across the HO sequences from RRR to AAA for FO alternations and repetitions (see Figure 1). In such a display, a typical cost-benefit pattern consists of increasing RTs across the HO sequences within the FO repetitions branch but decreasing RTs across the HO sequences within the FO alternation branch. That is, in the ANOVA, there should be an interaction of the linear trend of the $\mathrm{HO}$ factor with the FO factor, owing to an increasing slope for FO repetitions and a decreasing slope for FO alternations. In contrast, a cost-only pattern is characterized by positive slopes of the linear trends for both FO repetitions and FO alternations.

LRP onsets were measured using the jackknife-based procedure (Miller, Patterson, \& Ulrich, 1998; Ulrich \& Miller, 2001), which has been shown to increase the power in the analysis of LRP onsets. Ten different grand average LRPs for each of the experimental conditions were computed by omitting from each grand average the ERP data of another subject. LRP onsets were measured in the waveform of each grand average and were submitted to an ANOVA with $F$ values corrected as follows: $F_{\mathrm{C}}=F /(n-1)^{2}$, where $F_{\mathrm{C}}$ denotes the corrected $F$ value and $n$ the number of subjects (cf. Ulrich \& Miller, 2001).

Because of the marked LRP amplitude differences between HO sequences, we decided to use both a relative and two absolute criteria in order to measure the LRP onsets, as recommended by Miller et al. (1998). S-LRP onsets were measured relative to a 100-msec poststimulus baseline, using a relative criterion of $50 \%$ (onset is defined as when $50 \%$ of the maximal LRP amplitude has been reached) and two absolute criteria of $-0.6 \mu \mathrm{V}$ and $-0.8 \mu \mathrm{V}$. Effects in the LRP-R interval were obtained using the $50 \%$ relative criterion and two absolute criteria of $-0.8 \mu \mathrm{V}$ and $-1.0 \mu \mathrm{V}$. Here, a baseline of 100-msec duration, starting $300 \mathrm{msec}$ before response, was applied.

\section{Results}

Performance patterns. As is shown in Figure 1 (top), a typical cost-benefit pattern was found in RTs, statistically confirmed by a significant interaction of $\mathrm{HO}$ and FO stimulus sequence $[F(7,63)=22.1, p<.001]$ and a significant interaction of the linear trends for $\mathrm{FO}$ and $\mathrm{HO}$ $[F($ linear: 1,9$)=35.5, p<.001]$. Analyzing HO influences on FO repetitions and alternations separately, the repetition branch showed a positive slope, and the alter- 
nation branch showed a negative slope $\left[F_{\mathrm{s}}(\right.$ linear: 1,9$)=$ 27.8 and $36.4, p s<.001]$. There were no main effects for $\mathrm{FO}$ and $\mathrm{HO}$ sequences.

Fewer errors (Figure 1, middle) were made for FO repetitions, as compared with alternations $[M(\mathrm{R}$ vs. $\mathrm{A})=3.3 \%$ vs. $6.3 \% ; F(1,9)=21.9, p<.001]$. The HO sequences interacted with the FO stimulus sequences $[F(7,63)=27.0$, $p<.001 ; F($ linear: 1,9$)=87.0, p<.001]$ but showed no main effect.

Traces. The presence of residual activation traces was assessed by separating sequential effects in RTs according to the speed of the second-to-last response and by analyzing the long-term development of LRP amplitude across several trials. To test the influence of response speed in the previous trial on sequential patterns in the RT to the current stimulus, RTs from each condition and each subject were split into five bins of increasing RT, with an equal number of trials in each bin. ${ }^{3}$ The bin conditions are ordered from Bin 1, which included the $20 \%$ fastest preceding reactions for each condition, to Bin 5 , which included the $20 \%$ slowest preceding reactions $[M($ Bins $1-5)=242,277,299,325$, and $402 \mathrm{msec}$, respectively]. As is suggested by Figure 1 (bottom), for fast preceding reactions, the slopes of the two branches, especially the repetition branch, are steeper than those for slow preceding reactions, which was confirmed by a significant bin $\times \mathrm{FO} \times \mathrm{HO}$ interaction $[F(28,252)=5.0$, $p<.001]$. Testing the slopes of each of the two branches separately, significant bin $\times \mathrm{HO}$ interactions both for the repetition and the alternation branches $\left[F_{\mathrm{s}}(28,252)=3.8\right.$ and $2.5, p<.01]$ were found. There was also an increasing benefit for FO repetitions, as compared with alternations, with increasing speed of the preceding reactions $[F(4,36)=18.8, p<.001]$.

In order to study long-term development of lateralized motor activity preceding the terminal stimulus, recording epochs were analyzed, covering the current and the four preceding events $(-5,500$ to $1,000 \mathrm{msec})$. Because the type of final event is irrelevant for this kind of perspective, data were pooled over the FO sequence, with a change in the sign of activity for $\mathrm{FO}$ alternations. In order to emphasize the long-term trend, the data were low-pass filtered at $0.1 \mathrm{~Hz}$ and were referred to a $200-\mathrm{msec}$ baseline at the beginning of the long epoch (Figure 2). Apparently, there was a strong accumulation of lateralized activity as a function of the number of repetitions preceding the terminal event that was strongest over the $\mathrm{C} 3 / \mathrm{C} 4$ electrode sites. The accumulation was quantified as the average voltage within a 100 -msec interval preceding the final event and were submitted to an ANOVA, which confirmed the increasing amplitude with increasing number of preceding repetitions $[F(7,63)=22.8, p<$ $.001 ; F($ linear: 1,9$)=53.3, p<.001 ;$ Figure 2$]$.

Localization. Figure 3 shows the stimulus-locked LRPs (left-hand side) and the response-locked LRPs (righthand side) for the most extreme sequences ending with a repetition (upper LRPs) and with an alternation (lower
LRPs). The S-LRP interval revealed a significant costbenefit pattern (Figure 4), statistically confirmed by an interaction of the linear trends for $\mathrm{FO}$ and $\mathrm{HO}[F \mathrm{~s}$ (linear: $1,9)>8.2, p \mathrm{~s}<.05$ ] for all three onset criteria. When the HO linear trend was tested within each FO branch separately, it failed to reach significance for FO repetitions $[F($ linear: 1,9$)=2.6]$ for the $-0.8 \mu \mathrm{V}$ criterion but reached significance for both the relative and the $-0.6 \mu \mathrm{V}$ absolute criteria $[F \mathrm{~s}($ linear: 1,9$)>9.8, p \mathrm{~s}<.05]$. The alternation branch showed a significant negative slope for the relative and the $-0.8 \mu \mathrm{V}$ absolute criteria $\left[F_{\mathrm{s}}\right.$ (linear: $1,9)>14.0, p<.01]$ and a trend for this effect in the $-0.6 \mu \mathrm{V}$ criterion [ $F($ linear: 1,9$)=4.0, p<.1]$.

In the LRP-R interval, an interaction of the linear trends for $\mathrm{FO}$ and $\mathrm{HO}$ was present only for the absolute criterion and only as a trend $[F($ linear: 1,9$)>3.8, p<.1$; Figures 3 and 4]. When the HO slopes for the repetition and the alternation branches were tested separately, it was positive for the repetition branch-statistically verifiable, again, only when the absolute criterion was applied $[F($ linear: 1,9$)>4.0, p<.1]$ - but absent for the alternation branch $[F($ linear: 1,9$)<0.8]$.

\section{Discussion}

As has commonly been reported for RSIs greater than $500 \mathrm{msec}$, an HO cost-benefit pattern was observed in performance. Although an FO alternation effect was expected, the absence of any FO effect in long-RSI conditions is not altogether unusual (e.g., Sommer et al., 1999). As regards the trace mechanism, the RTs were indeed differentially related to the speed of the previous responsethat is, to the ITI. The HO cost-benefit pattern was more pronounced for short ITIs, and there was also a tendency toward an FO repetition effect for the short RT bins- that is, for short ITIs. These findings are broadly in line with a passively decaying activation trace. More specific evidence was obtained over four trials from the long-term LRP amplitude, which increased with the number of repetitions preceding the current stimulus. This observation might be considered as an index of the accumulation of response-related traces across response repetitions.

The functional localization of sequential effects in the information processing system was undertaken by measuring the $\mathrm{S}-\mathrm{LRP}$ onset and the LRP-R intervals as chronometrical markers of loci of action before or after the start of response-related motor activation, respectively. The S-LRP interval suggested that the cost-benefit pattern in performance with opposite slopes for the repetition and the alternation branches had a primarily premotoric locus of origin. The LRP-R interval seemed to decrease when an increasing number of repetitions preceded a final repetition, indicating a motoric locus for the increasing RT within the FO repetition branch. In contrast, no HO effects were seen in the alternation branch for the LRP-R. Together, these results indicate that the locus of the cost-benefit pattern, with its positive slope for the RTs for the repetition branch and a neg- 

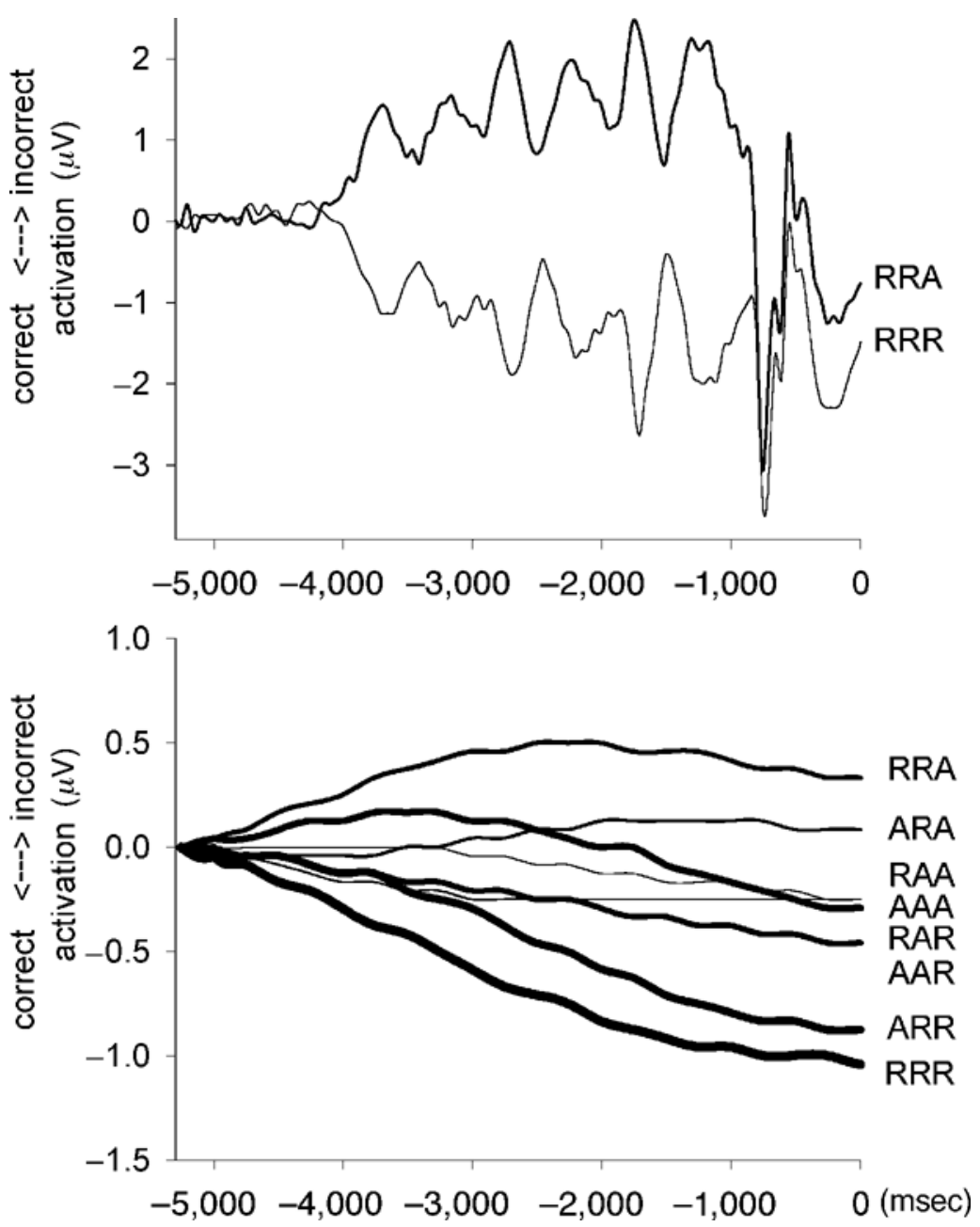

Figure 2. Experiment 1, top: development of long-term lateralized readiness potential (LRP) activity for the higher order sequences RRA and RRR across the 5.5 sec preceding the current stimulus onset at $t=0$. Bottom: long-term LRP for all eight higher order sequences, low-pass filtering at $0.1 \mathrm{~Hz}$. The conditions are averaged over first-order sequences, with a change in the sign of activity for first-order alternations.

ative slope for the RTs for the alternation branch (the hallmark of the cost-benefit pattern), is wholly premotoric in origin. In addition, the positive slope of the FO repetition branch appears to have a motoric source as well. The motoric source may stem from the motoric trace accumulation observed in the long-term LRP amplitude.

\section{EXPERIMENT 2}

This experiment was designed to yield a cost-only pattern in performance by using very short RSIs in the same compatible arrangement as that in Experiment 1. If passive but specific traces contribute to sequential effects also, when the cost-only pattern is present, they should be even stronger in this experiment than in the long-RSI condition of the previous experiment, because of the strongly diminished ITI. Remember, the cost-only pat- tern itself cannot be explained by a trace concept, and so additional mechanisms were expected to be present in this experiment.

\section{Method}

Ten subjects (4 males), between 19 and 38 years of age (mean, 27.4 years), were tested. All were strongly right-handed, with handedness quotients greater than +60. Except for an RSI of $50 \mathrm{msec}$, the method was the same as that in Experiment 1.

\section{Results}

Performance patterns. Figure 5 (top) shows a clear cost-only pattern in RTs, statistically confirmed by a significant main effect of $\mathrm{HO}$ stimulus sequence $[F(7,63)=$ $20.0, p<.001]$. This holds true also for the positively sloped linear trend across the HO sequences as a main effect $[F($ linear: 1,9$)=23.5, p<.001]$ and also for the rep- 


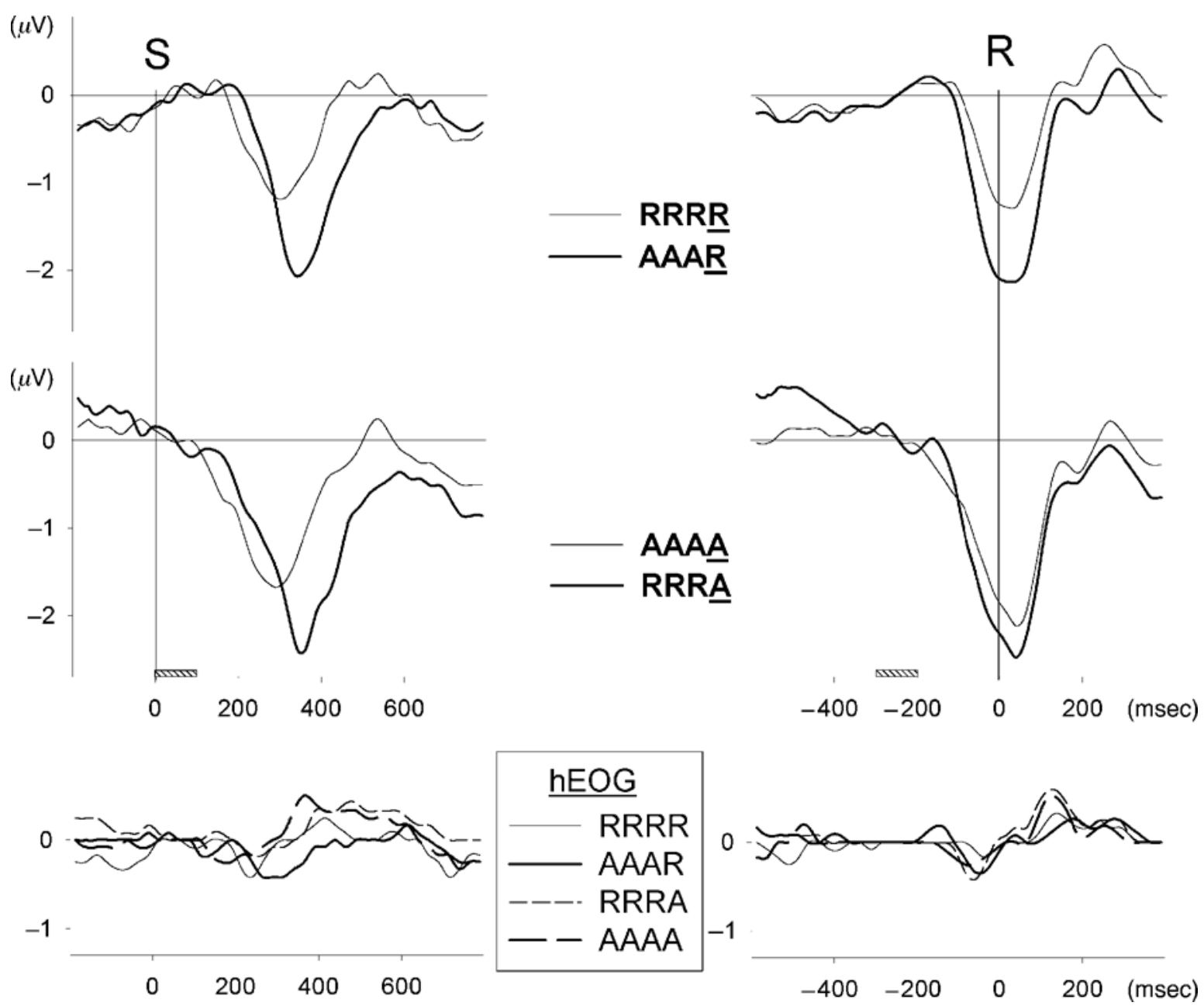

Figure 3. Experiment 1: grand mean lateralized readiness potential (LRP) and hEOG waveforms for the extreme sequences RRRR and AAAR of the repetition branch (upper LRPs) and for the extreme sequences AAAA and RRRA of the alternation branch (lower LRPs). The left and right panels show the stimulus- and response-synchronized LRPs (S-LRP and LRP-R), respectively.

etition and the alternation branches separately $[F \mathrm{~s}($ linear: $1,9)=34.0$ and $11.8, p s<.01]$. There was no FO effect in RTs.

Fewer errors were made for FO repetitions, as compared with alternations $[M(\mathrm{R}$ vs. $\mathrm{A})=4.8 \%$ vs. $12.7 \%$; $F(1,9)=11.6, p<.01 ;$ Figure 5 , middle $]$. There was a main effect of HO sequences $[F(7,63)=16.2, p<.001]$, with error rates increasing monotonically from RRR to AAA $[F($ linear: 1,9$)=30.4, p<.001]$.

Traces. As before, the sequential effects in RTs were studied as a function of the speed of the second-to-last response by considering the five RT bins $[M($ Bins $1-5)=$ $354,402,434,473$, and $572 \mathrm{msec}$, respectively]. Figure 5 (bottom) shows that for fast preceding responses, the slopes of the repetition and the alternation branches were differentially affected, with a steeper positive slope in the repetition branch and a smaller positive slope in the alternation branch, as compared with slow preceding re- sponse bins, confirmed by a significant bin $\times \mathrm{FO} \times \mathrm{HO}$ interaction $[F(28,252)=4.0, p<.001]$. Testing the two branches separately, an ANOVA confirmed that the HO slope for FO repetitions decreased with increasing preceding RTs and increased for FO alternations $\left[F_{\mathrm{S}}(14,126)=\right.$ 3.9 vs. $1.8, p$ s < .05; see also Table 1]. As was already observed in Experiment 1, there was a stronger FO repetition effect for fast than for slow preceding responses $[F(4,36)=4.7, p<.01]$, although the FO repetition effect itself was not significant in either bin condition.

As before, four preceding events and the current event were included in the analysis of long-term LRP amplitude ( $-3,000$ to $800 \mathrm{msec})$, filtered, baseline-corrected, and quantified as above. Again, LRP amplitude increased with the number of repetitions preceding the current stimulus $[F(7,63)=12.0, p<.001 ; F($ linear: 1,9$)=$ $79.7, p<.001]$, being largest for continued repetitions (Figure 6). 


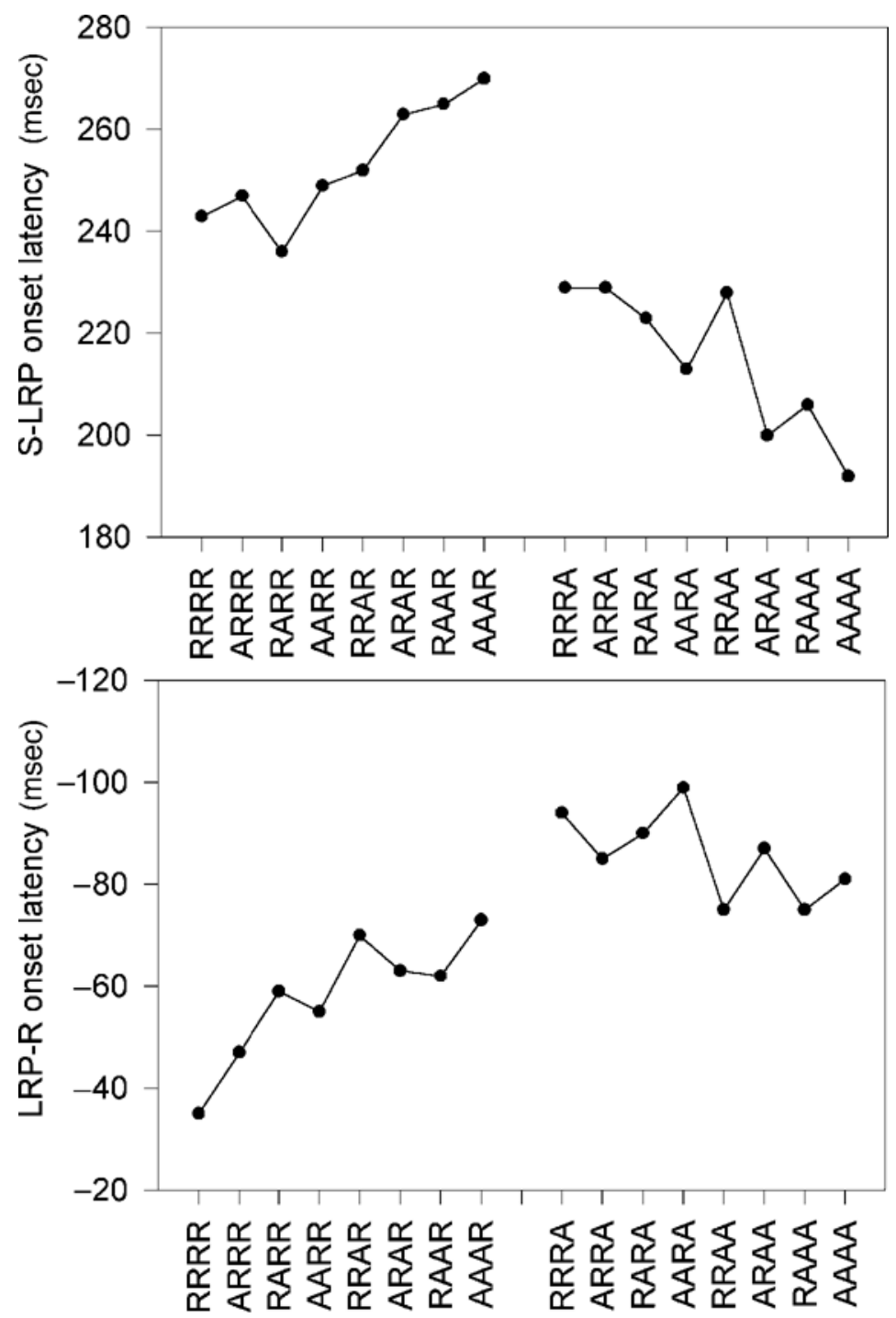

Figure 4. Experiment 1: sequential effects in the stimulus to lateralized readiness potential (S-LRP) and LRP to response (LRP-R) onset latencies (top vs. bottom) intervals, using the absolute $-0.8 \mu \mathrm{V}$ onset criterion.

Localization. Because of massive baseline problems owing to the short interval between successive trials, it was not possible in this experiment to derive reliable LRP onset values.

\section{Discussion}

At the performance level, a clear cost-only pattern emerged both in RTs and in error rates. The absence of an FO repetition effect in the RTs of the present experiment might be due to a speed-accuracy tradeoff, because error rates revealed a strong repetition effect.

Confirming the results of Experiment 1, sequential effects in RTs were related to the speed of the previous responses. An increasing benefit for FO repetitions with increasing prior response speed was observed. Moreover, the influence of a cost-benefit pattern seemed to increase with increasing prior speed. That is, after fast preceding responses, the slope of the FO repetition branch was more positive, and that of the alternation branch was less positive after fast than after slow preceding responses. Thus, the observed cost-only pattern seems to be shifted toward a cost-benefit pattern for fast preceded reactions-that is, for shorter ITIs. Possible contributions of processing traces were also indicated by the analysis of long-term LRP amplitudes, which, by and large, showed a pattern similar to that in Experiment 1. A more formal comparison of the long-term LRP patterns will be presented in the General Discussion section.

However, it may be an observation of some relevance for the mechanisms underlying the cost-only effect that 
Table 1

Reaction Time (RT) Bin Analysis for All Three Experiments

\begin{tabular}{|c|c|c|c|c|c|c|c|c|c|c|c|c|}
\hline \multirow[b]{3}{*}{ Bin } & \multicolumn{4}{|c|}{ Experiment 1} & \multicolumn{4}{|c|}{ Experiment 2} & \multicolumn{4}{|c|}{ Experiment 3} \\
\hline & \multicolumn{2}{|c|}{$\begin{array}{l}\text { Repetition } \\
\text { Branch }\end{array}$} & \multicolumn{2}{|c|}{$\begin{array}{c}\text { Alternation } \\
\text { Branch }\end{array}$} & \multicolumn{2}{|c|}{$\begin{array}{l}\text { Repetition } \\
\text { Branch }\end{array}$} & \multicolumn{2}{|c|}{$\begin{array}{c}\text { Alternation } \\
\text { Branch }\end{array}$} & \multicolumn{2}{|c|}{$\begin{array}{l}\text { Repetition } \\
\text { Branch }\end{array}$} & \multicolumn{2}{|c|}{$\begin{array}{c}\text { Alternation } \\
\text { Branch }\end{array}$} \\
\hline & Slope & $\mathrm{RT}$ & Slope & RT & Slope & $\mathrm{RT}$ & Slope & RT & Slope & $\mathrm{RT}$ & Slope & $\mathrm{RT}$ \\
\hline 1 & 10.2 & 291 & -10.7 & 303 & 13.0 & 411 & 6.2 & 424 & 11.2 & 331 & -4.3 & 392 \\
\hline 2 & 8.6 & 300 & -9.5 & 304 & 10.4 & 428 & 7.3 & 431 & 11.2 & 346 & -2.5 & 399 \\
\hline 3 & 7.8 & 309 & -9.0 & 305 & 8.2 & 436 & 9.4 & 442 & 10.0 & 355 & -3.1 & 402 \\
\hline 4 & 7.2 & 312 & -8.6 & 307 & 7.6 & 456 & 10.0 & 455 & 10.3 & 366 & -2.4 & 408 \\
\hline 5 & 5.4 & 319 & -6.1 & 311 & 4.8 & 484 & 8.7 & 478 & 7.0 & 374 & -1.4 & 417 \\
\hline
\end{tabular}

Note-The table shows the slopes of the repetition and the alternation branches, together with the mean RTs (in milliseconds) of the current responses for the two first-order sequences for each of the five RT bins.

this pattern was not enhanced in the short preceding-RT bin, being statistically confirmed by the missing interaction of bin with $\mathrm{HO}$ sequence $(p<.1)$. This finding may indicate that the main factor causing processing costs that are due to alternations or benefits that are due to repetitions is more tightly synchronized to responserelated processes than to early processes in the preceding trial.

\section{EXPERIMENT 3}

This experiment was conducted to examine the locus and mechanisms of sequential patterns in a long-RSI condition, but with a less compatible stimulus-to-response mapping than that in Experiment 1. In noncompatible mappings, the cost-benefit pattern is weaker, and repetition benefits increase. These findings are normally explained by a stronger influence of cost-only related mechanisms in less compatible experiments. Therefore, one should find, in the present experiment, both costonly and cost-benefit influences on RT patterns, which might allow for the differential localization of sequential contributions. In addition, the aim of the experiment was to replicate the findings of the first two experiments concerning trace activity.

\section{Method}

A total of 10 subjects ( 4 males), between 20 and 28 years of age (mean, 24.4 years), were tested in this experiment. They were strongly right-handed, with handedness quotients greater than +60 . Stimuli were red or green dots (diameter, $5 \mathrm{~mm}$ ) appearing always in the center of the monitor, where a fixation point also was continuously present even during stimulus presentation. Each color was assigned to one of the keys. The assignments of finger to key and of key to color were counterbalanced over subjects. Otherwise, the method was the same as that in Experiment 1, where also an RSI of $700 \mathrm{msec}$ was applied.

\section{Results}

Performance. Figure 7 (top) displays a pattern in RTs in which a pronounced positive slope for the repetition branch contrasts with a weak negative slope in the alternation branch. An ANOVA yielded a significant interaction of $\mathrm{HO}$ and $\mathrm{FO}$ stimulus sequence $[F(7,63)=27.7$, $p<.001 ; F($ linear: 1,9$)=55.6, p<.001]$ and a main effect of the HO sequence $[F(7,63)=14.5, p<.001 ; F$ (linear: $1,9)=20.6, p<.01]$. The positive slope in the repetition branch was significant, whereas the negative slope in the alternation branch was only a trend $[F \mathrm{~s}($ linear: 1,9$)=$ 94.9 and $4.8, p s<.001$ and .06 , respectively]. These effects were accompanied by an FO repetition effect-that is, faster reactions for repetitions than for alternations $[M(\mathrm{R}$ vs. $\mathrm{A})=354$ vs. $404 \mathrm{msec} ; F(1,9)=83.3, p<.001]$.

Fewer errors (Figure 7, middle) were made for FO repetitions than for alternations $[M=4.2 \%$ vs. $7.2 \% ; F(1,9)=$ $10.4, p<.01]$. The $\mathrm{HO}$ sequence affected error rates as a main effect $[F(7,63)=8.0, p<.001 ; F($ linear: 1,9$)=$ $15.9, p<.01]$ and also in interaction with the FO stimulus sequence $[F(7,63)=13.1, p<.001 ; F($ linear: 1,9$)=$ $27.3, p<.001]$.

Traces. As before, sequential effects were tested for separate RT bins as a function of response speed in the preceding trial $[M($ Bins $1-5)=298,341,369,398$, and $498 \mathrm{msec}$, respectively]. Consistent with the other experiments, preceding response speed differentially affected the slopes of the repetition and the alternation branches, which was confirmed by a significant bin $X$ FO $\times$ HO interaction $[F(28,252)=3.4, p<.001$; Figure 7 , bottom]. Testing the two branches separately, the repetition branch was steeper for fast preceded reactions $[F(28,252)=3.3, p<.001]$. The slope in the alternation branch appeared to be somewhat more negative in the fast bins, which was not significant, however $[F(28,252)=$ $1.1, p>.1]$. As in the previous experiments, there was also a stronger repetition effect for fast than for slow preceding reactions $[F(4,36)=6.7, p<.01]$.

The analysis of long-term LRP amplitude was performed in the same way as for Experiment 1. Again, there was an increase in LRP negativity with the number of preceding repetitions $[F(7,63)=2.0, p<.1 ; F$ (linear: $1,9)=9.1, p<.05 ;$ Figure 8$]$.

Localization. Both S-LRP and LRP-R intervals were measured as in Experiment 1 (Figures 9 and 10). In the S-LRP interval, there was a significant main effect of HO sequences [ $F($ linear: 1,9$)=11.7, p<.01$ ], but only for the $-0.8 \mu \mathrm{V}$ absolute criterion. Whereas the repetition branch alone showed a significant positive linear 


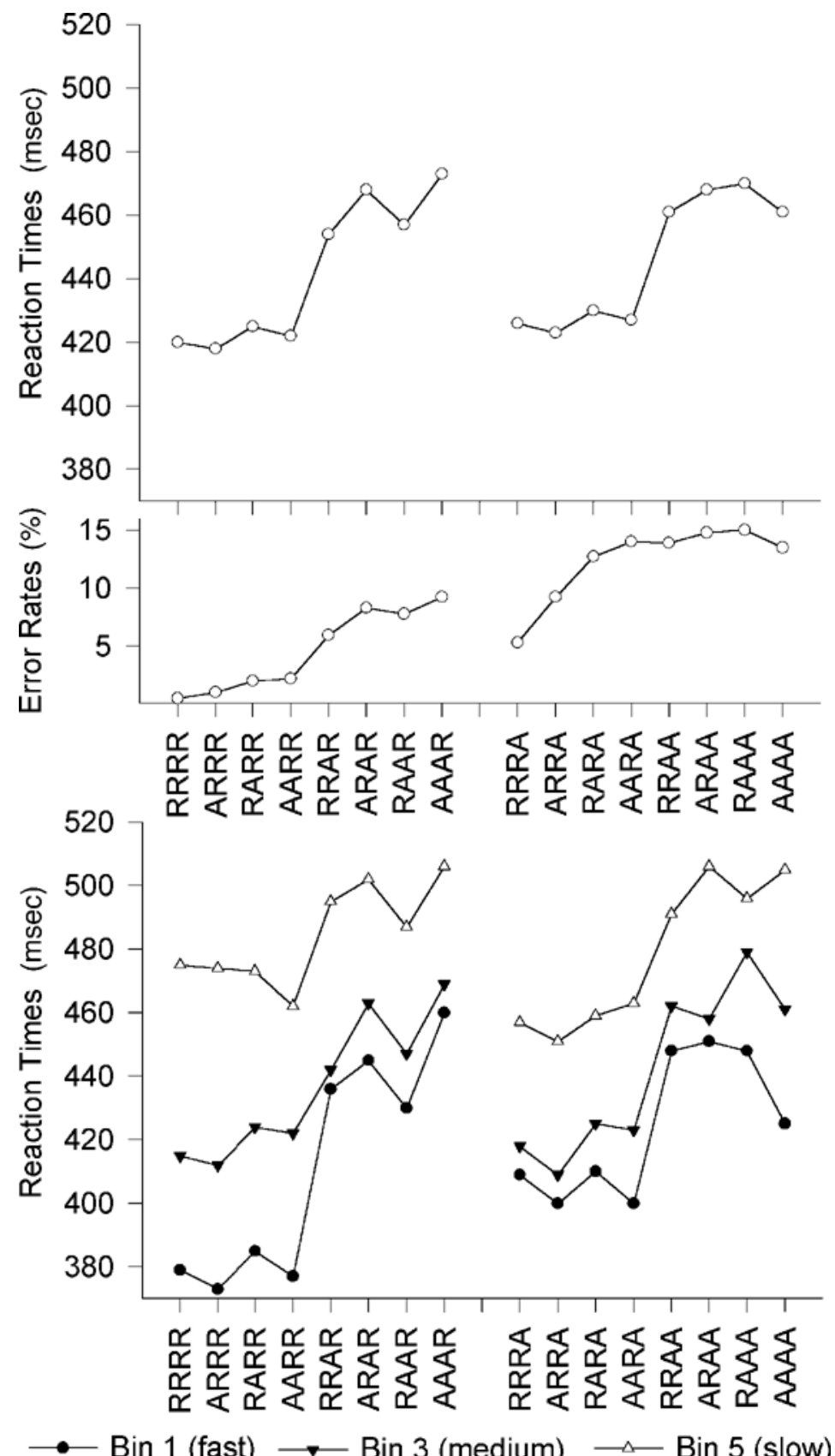

Figure 5. Experiment 2: sequential effects in mean reaction times (RTs; top), in mean error rates (middle), and in RTs partitioned according to the speed of the preceding response (bottom; mean RTs of the fastest, the slowest, and the middle of five equal bins).

trend across the HO sequences for all three onset criteria $[F$ s(linear: 1,9$)>7.1, p<.05]$, the alternation branch showed a significant linear trend only for the relative onset criterion [ $F($ linear: 1,9$)=17.5, p>.01]$.

The LRP-R interval showed a significant interaction of the linear trends for $\mathrm{FO}$ and $\mathrm{HO}$ for all three onset criteria $\left[F_{\mathrm{s}}(\right.$ linear: 1,9$\left.)>6.0, p<.05\right]$. Also, for all three criteria, the linear trend across the $\mathrm{HO}$ sequences was significant for the repetition branch alone $[F \mathrm{~s}($ linear: 1,9$)>6.2, p<.05]$, but not for the alternation branch $\left[F_{\mathrm{s}}(\right.$ linear: 1,9$\left.)<1.8\right]$.

\section{Discussion}

As was expected, the performance pattern in this experiment suggested a mixture of both cost-benefit and 


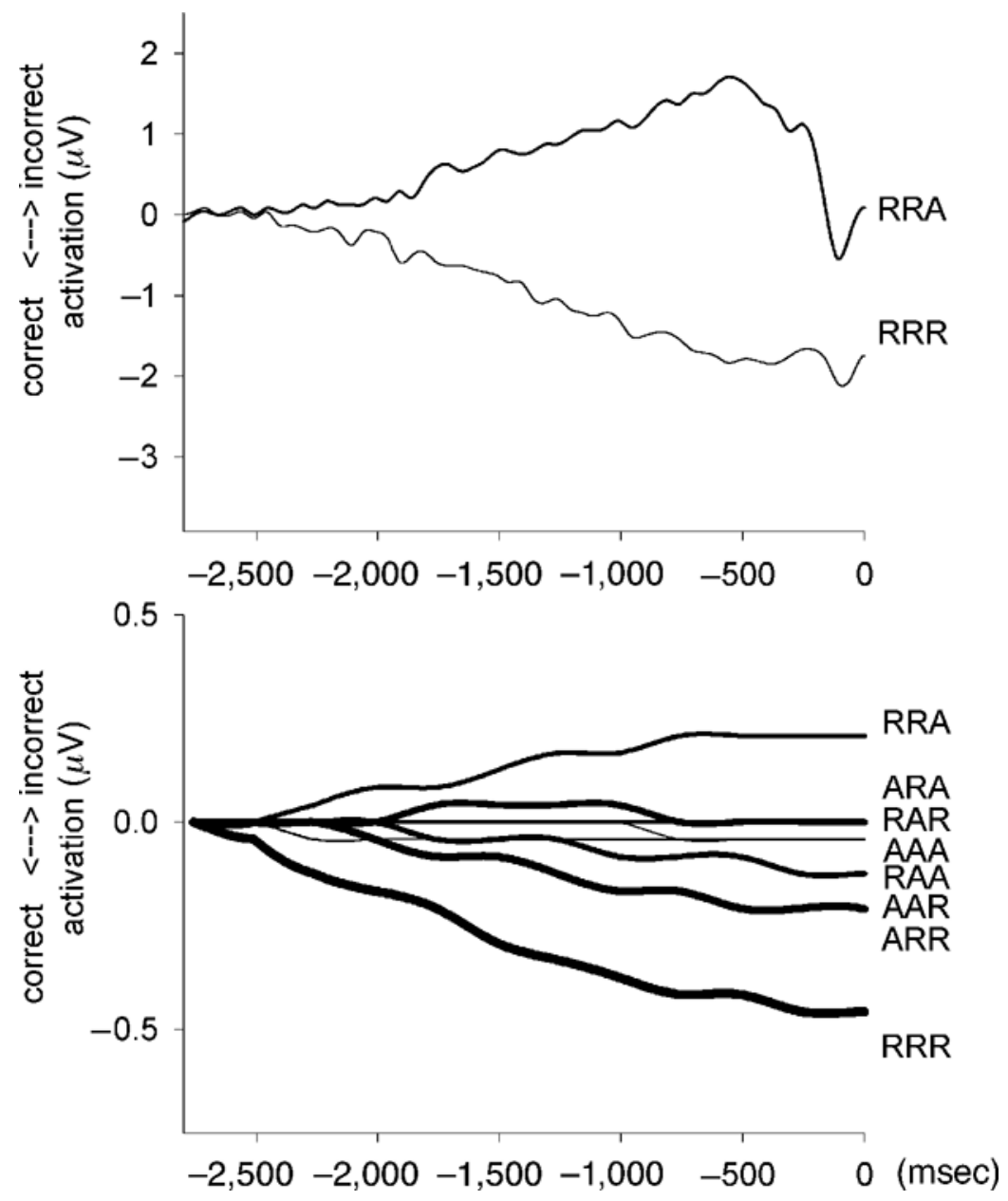

Figure 6. Experiment 2, top: development of long-term lateralized readiness potential (LRP) activity for the higher order sequences RRR and RRA across the 5.5 sec preceding the current stimulus onset at $t=0$. Bottom: long-term LRP for all eight higher order sequences, low-pass filtering at $0.1 \mathrm{~Hz}$.

cost-only patterns, presumably owing to the noncompatible stimulus-response mapping. A positive slope of RTs across the FO repetition branch was combined with a very small negative slope for the alternation branch.

Consistent with the other experiments, both $\mathrm{HO}$ and FO sequences were influenced by response speed in the previous trial, with faster preceding reactions producing larger repetition benefits, as well as an increased cost-benefit pattern, in the HO sequences. Supporting trace accounts, a clear accumulation of hand-specific lateralized activity was observed with an increasing number of event repetitions.

It was of particular interest here whether the small slope for the alternation branch in performance might be due to opposing tendencies of cost-benefit and cost-only in motoric and premotoric stages or merely to diminished effects at both levels. The positive slope in processing time for the repetition branch obviously has two sources. One source is motoric-as shown in the LRP-R interval - and might be related to motoric trace mechanisms. The second source is premotoric according to the S-LRP interval. Both the premotoric and the motoric sources act synergistically to decrease processing time as the number of repetitions preceding the current stimulus increases. As to the alternation branch, it appears that there are no clear sequential effects at any level. Therefore, the small negative slope in the RTs is not due to the canceling out of opposing effects at stimulus- and response-related stages. In contrast, from the findings of Experiment 1 and the missing effect for the alternation branch in Experiment 3, one might conclude that both the cost-only and the cost-benefit patterns have a mainly premotoric origin. The suggested overlap of both mechanisms in this experiment might explain the missing negative alternation slope in the S-LRP interval, in contrast to a remarkable effect in the first experiment. This hypothesis has to be tested more directly in the future. 

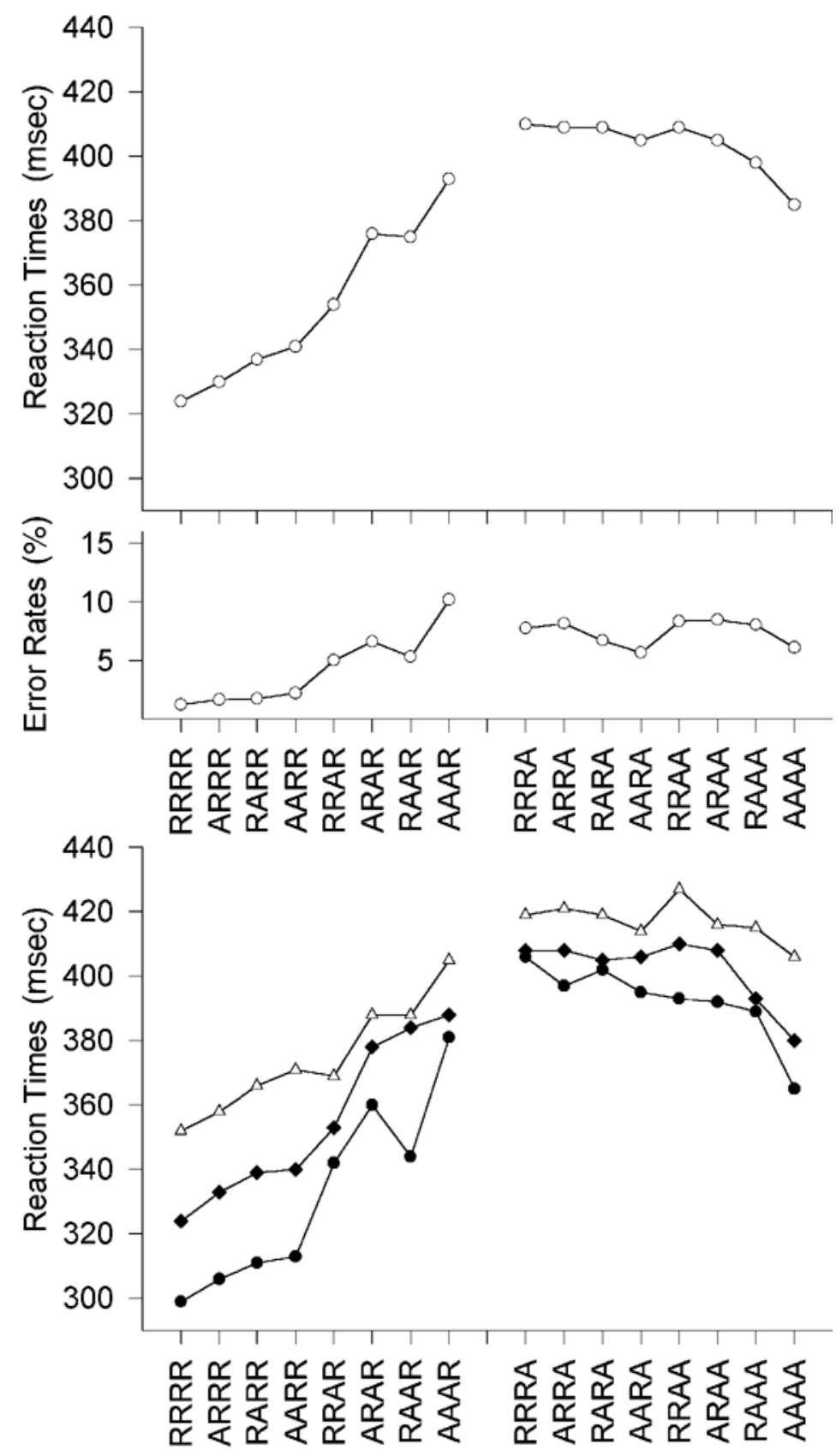

$\longrightarrow \operatorname{Bin} 1$ (fast) $\longrightarrow$ Bin 3 (medium) $\longleftarrow$ Bin 5 (slow)

Figure 7. Experiment 3: sequential effects in mean reaction times (RTs; top), in mean error rates (middle), and in RTs partitioned according to the speed of the preceding response (bottom; mean RTs of the fastest, the slowest, and the middle of five equal bins).

\section{GENERAL DISCUSSION}

Primary objectives of the present paper were the search for trace accumulation as a basic mechanism responsible for sequential effects and the localization of these effects within the information-processing system.
First, the performance results will be summarized, followed, in turn, by a discussion of these two objectives.

\section{Performance Patterns}

The three experiments yielded the expected HO sequential patterns in performance. Conforming with nu- 


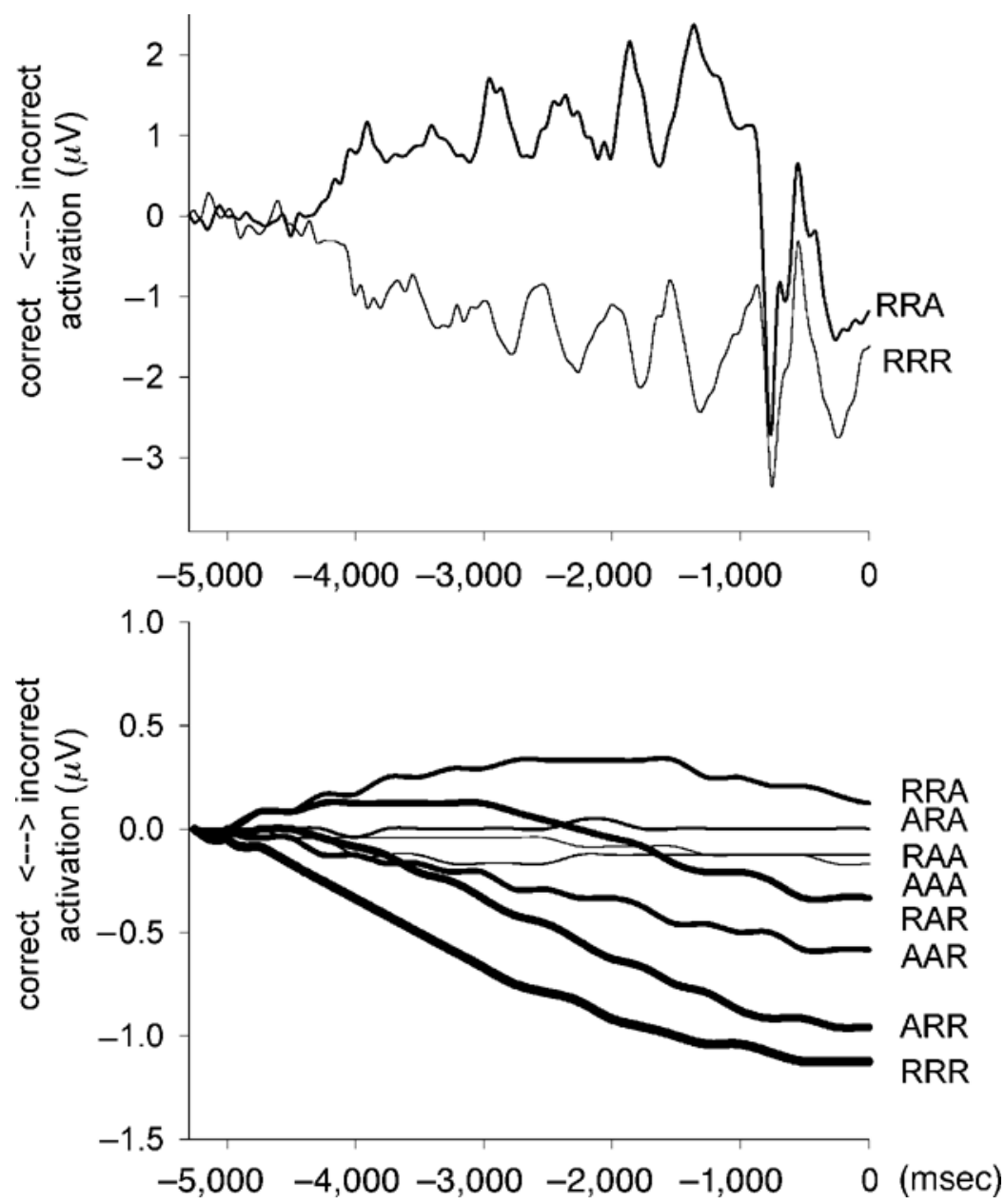

Figure 8. Experiment 3, top: development of long-term lateralized readiness potential (LRP) activity for the higher order sequences RRR and RRA across the 5.5 sec preceding the current stimulus onset at $t=0$. Bottom: long-term LRP for all eight higher order sequences, low-pass filtering at $0.1 \mathrm{~Hz}$.

merous findings in compatible stimulus-to-response assignments, a cost-benefit pattern was observed at long RSIs (Experiment 1), whereas at short RSIs (Experiment 2), the pattern was cost-only. A noncompatible stimulus-response assignment at long RSIs (Experiment 3 ) produced a cost-benefit pattern in which the repetition branch was at least as strong as in compatible arrangements (Experiment 1) but the negative slope of the alternation branch was much reduced. Therefore, this pattern gave the expected impression of a mixture between cost-only and cost-benefit.

Confirming the separability of HO cost-benefit patterns and FO alternation effects, Experiment 1 did not yield an FO effect despite the strong cost-benefit pattern. Similarly, there was no FO repetition effect despite a clear HO cost-only effect in Experiment 2. The FO repetition effect in Experiment 3 appears to have been merely a consequence of the HO patterns, with short RTs for some $\mathrm{HO}$ sequences that were terminated by FO rep- etitions but relatively long RTs for all sequences terminated by alternations.

\section{Trace Accumulation}

Theoretical models of sequential effects posit that specific traces left from previous processing cycles are a major source of the cost-benefit pattern (e.g., Laming, 1969); but such traces are also incorporated as elements necessary to model cost-only patterns (Soetens et al., 1984). However, there is some dispute as to whether these traces passively decay over time or whether they merely depend on the succession of events. Two approaches were used in order to seek evidence for traces of residual activation.

First, the relationship between the ITI preceding the current event and the sequential pattern displayed by the RT to the current event was assessed by partitioning current RTs according to the speed of the preceding response. When stimulus presentation depends on the interval fol- 

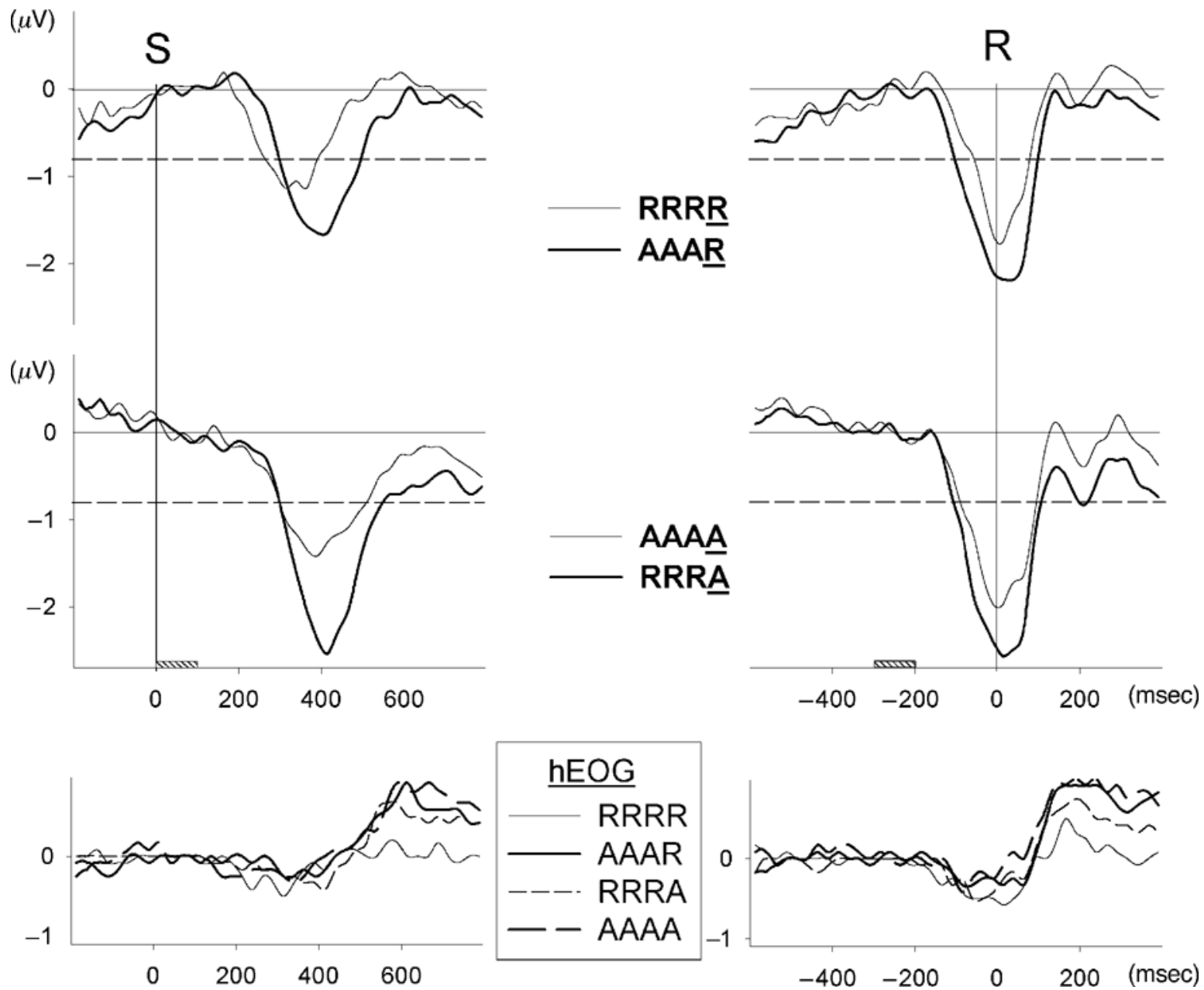

Figure 9. Experiment 3: grand mean lateralized readiness potential (LRP) and hEOG waveforms for the extreme sequences RRRR and AAAR of the repetition branch (upper LRPs) and for the extreme sequences RRRA and AAAA (lower LRPs) of the alternation branch. The left and right panels show the stimulus- and response-synchronized LRPs (S-LRP and LRP-R), respectively.

lowing the response (RSI), as was the case in the present experiments, the ITI is a direct function of the preceding RT. If passive traces are responsible for cost-benefit aspects in performance, this aspect should be stronger for the short than for the long preceding RT bin.

This prediction was borne out in all three experiments reported here (see Table 1). For short ITIs-that is, short preceding RTs - the cost-benefit aspect in the sequential effects was indeed consistently stronger than that for long ITIs over experiments, which was confirmed by a significant interaction of bin with FO and HO. In Experiment 1 , the pure cost-benefit pattern in overall RTs was much enhanced in the short preceding RT bin. In Experiment 2, with its overall cost-only pattern, the positive slope of the RTs in the FO repetition branch was enhanced in the short preceding RT bin, whereas the positive slope in the FO alternation branch tended to be diminished. That is, the cost-only pattern appeared to be shifted toward a cost-benefit pattern for fast preceded responses. Finally, in Experiment 3, the balance between cost-only and cost-benefit appeared to be shifted toward cost-only in the long preceding RT bin, but toward cost-benefit in the short RT bin. Overall, these findings indicate that the cost-benefit contributions to any kind of sequential pattern are stronger if the interval between successive trials is shortened. Although correlational, these results support a passive trace account of the cost-benefit pattern. Moreover, the findings also indicate that even when performance shows pure cost-only, as in the case of Experiment 2, contributions of costbenefit producing mechanisms are present and can be detached by suitable measures. Interestingly, the second hypothetical predictor for a trace account, an increase in the FO repetition effect with a decrease in the ITI, could be seen in all three experiments.

A second indicator for the contributions of residual traces to sequential effects was the amplitude of the LRP analyzed across several trials. This indicator is more 


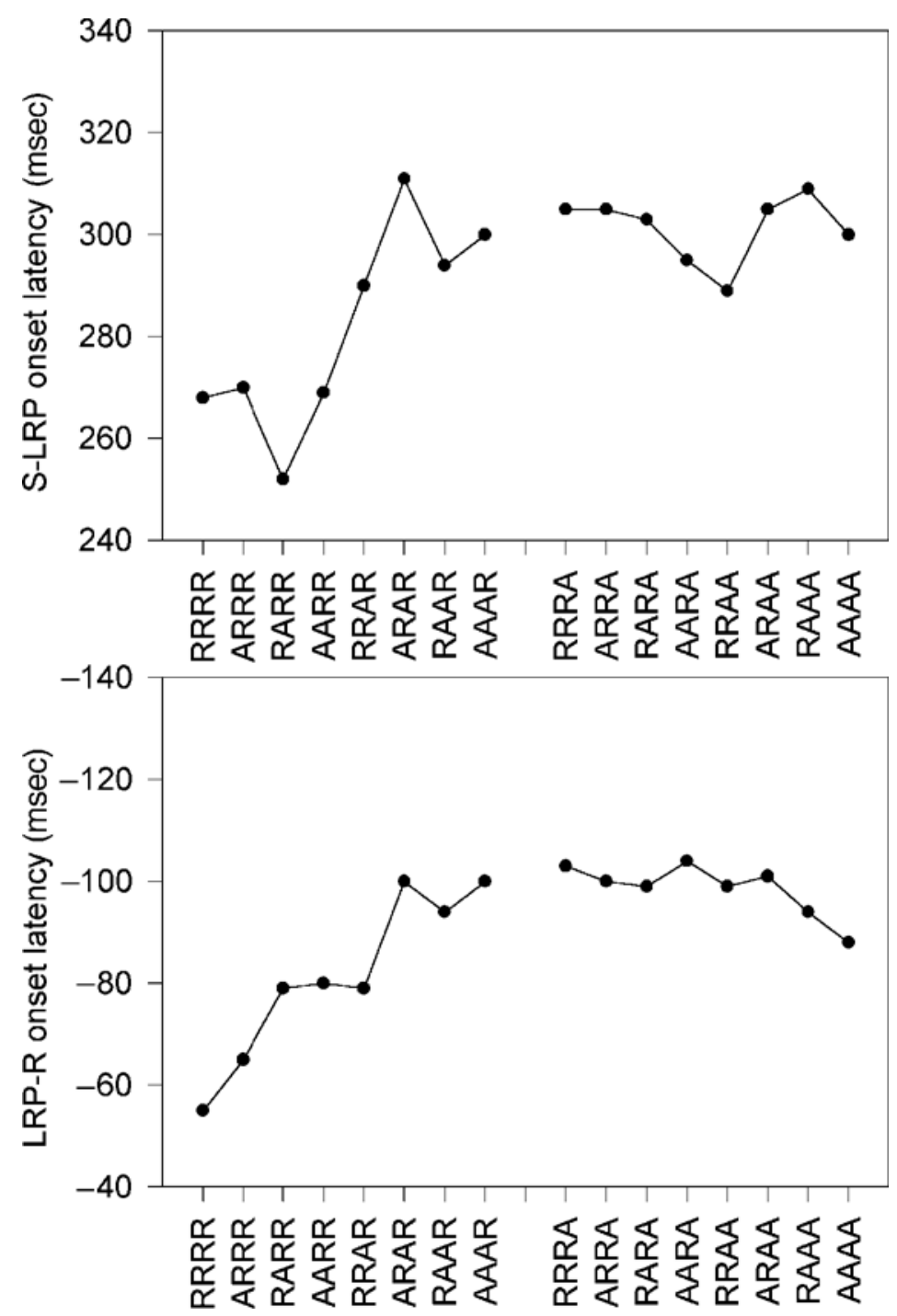

Figure 10. Experiment 3: sequential effects in the stimulus to lateralized readiness potential (S-LRP) and LRP to response (LRP-R) onset latencies (top vs. bottom) intervals, using the absolute $-0.8 \mu \mathrm{V}$ onset criterion.

specific than RT partitioning, because it reflects handspecific response activation only and does not show residual traces of premotoric activation. The long-term LRP recordings show that activation following a response does not return to baseline by the time the next stimulus is presented, even at long RSIs. Rather, it indicates that response activation in the current trial is superimposed on residual activation left from previous trials, especially if the current response is the same as the preceding one. When there are several such repetitions in a row, the activation appears to be cumulative. From this analysis, it is clear that there is motoric activation prior to the current stimulus in some conditions. It is plausible to assume that preexisting motoric activation is facilitative to a response when it is in accord with activation.

In order to obtain a clearer picture of the rules governing the residual activation reflected in long-term LRP, a mathematical model of passive, decaying traces was applied to the amplitude measures obtained in the three experiments. In this model, the passive accumulation $T_{(\mathrm{A}-\mathrm{B}), N}$ of activity on trial $N$ for the occurrence of Stimulus A relative to the occurrence of $\mathrm{B}$ was defined as follows:

$$
T_{(A-B), N}=w \sum_{i=N-1}^{1}\left(\alpha^{\mathrm{ISI}}\right)^{N-i} S_{i},
$$

with

$$
S_{i}=\frac{-1 \text { for }\left(S_{i}=\mathrm{B}\right)}{1 \text { for }\left(S_{i}=\mathrm{A}\right)} .
$$

This formulation includes two independent parameters, the decay rate $\alpha$ and a weighting factor $w$ of an exponential decay function. The decay rate $\alpha$ expresses the amount of residual activity relative to the maximal activation left at the moment when the next stimulus is pre- 
Table 2

Goodness of Fit for the Model of Passive Trace Accumulation Applied to the Long-Term Accumulation of Lateralized Readiness Potential Amplitudes for All Three Experiments

\begin{tabular}{|c|c|c|c|c|c|}
\hline \multirow[b]{2}{*}{ Experiment } & \multirow[b]{2}{*}{ ISI (sec) } & \multicolumn{2}{|c|}{ Parameters } & \multirow[b]{2}{*}{$S E$} & \multirow[b]{2}{*}{$\% \mathrm{Var}$} \\
\hline & & $\alpha$ & $w$ & & \\
\hline 1 & 1.009 & 0.73 & 0.53 & 0.36 & 94.5 \\
\hline 2 & 0.494 & 0.74 & 0.15 & 0.22 & 86.8 \\
\hline 3 & 1.049 & 0.68 & 0.66 & 0.42 & 93.4 \\
\hline
\end{tabular}

Note- $S E$, standard error of estimate in $\mu \mathrm{V} ; \%$ var, percentage of variance accounted for

sented. Because the interstimulus interval (ISI) was included as a constant of the model, the decay rate can be directly compared across the experiments as a measure of decay rates. For present purposes, the mean ISI in the three experiments was approximated as the sum of mean RTs over conditions and RSI intervals.

Table 2 gives the results of a least-square fit of the model parameters to the long-term LRP amplitudes. Overall, the fit of the decaying trace model with the data was satisfying, with residual variances between about $5 \%$ and $15 \%$. Interestingly, the ISI-corrected decay rates were very similar across all three experiments despite very different RSIs (Experiment 1 vs. Experiment 2) and different degrees of stimulus-response compatibility (Experiment 1 vs. Experiment 3). The main difference in long-term LRP activation between the experiments appears to consist in the weighting factor $w$, which was much reduced in Experiment 2. The weighting factor expresses maximum activation, or in other words, overall LRP amplitude (see Figure 11).

The overall amplitude reduction in the short-RSI experiment does not concern only the long-term activation but is also very conspicuous in the LRP amplitude of short one-trial epochs. To our knowledge, the decline in LRP amplitude in short-RSI (or short-ITI) conditions has not been reported before and may be of methodological relevance. Possibly, both the long- and the shortterm reductions of LRP amplitude relate to the same mechanism. On the assumption that there is an upper limit to cortical activation, it is conceivable that at short RSIs, activation of both motor cortices approaches saturation because of progressive activation accumulation.

\section{Localization of Sequential Effects Within Information Processing}

The second major objective of the present experiments was the localization of sequential effects within the information-processing system. This objective was
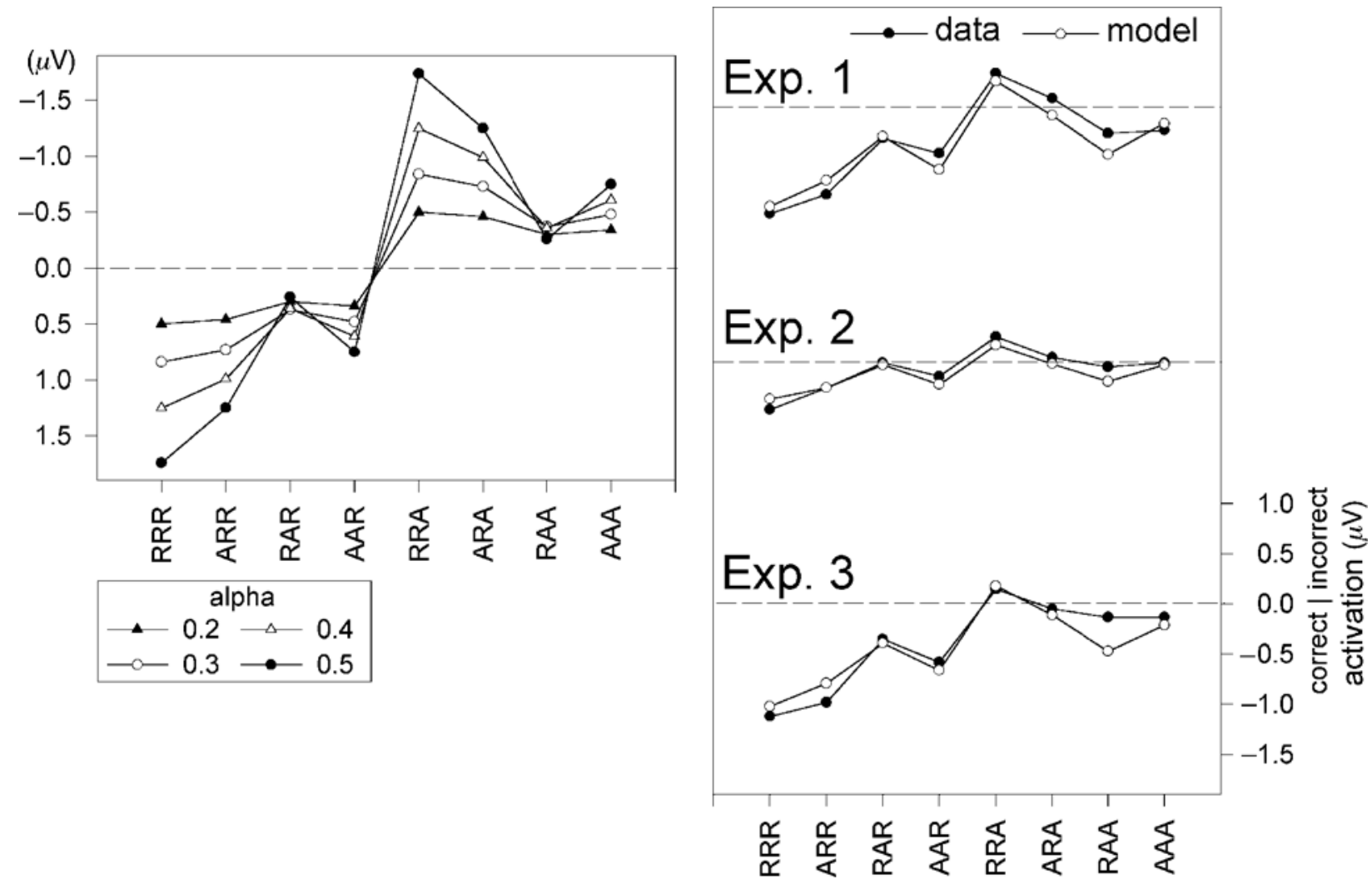

Figure 11. Left panel: theoretical predictions for sequential effects produced by the trace mechanism. The figure shows the influence of different decay rates alpha on the size of sequential patterns. In the model presented, a weighting factor of $w=2$ was chosen. Right panel: sequential effects of lateralized activity accumulated over four trials back before actual stimulus presentation. The figure shows the data for the three experiments, together with the fit to the trace model. The fitting parameters are shown in Table 2 . 
pursued by measuring the S-LRP and the LRP-R intervals as chronometrical markers of premotoric and motoric loci of action, respectively. As was already mentioned, FO effects in the LRP onsets of the present study have not been examined, because of the problem of defining an adequate baseline. As has been shown in the long-term LRP analysis, LRP activation does not settle between trials. Therefore, there will be differential baseline activation if trials are separated for different types of preceding stimuli, as is inevitable in the analysis of any kind of sequential effects. This problem is most serious when a prestimulus baseline is used for the assessment of FO effects; it is much less serious when comparisons are made within trials of the same FO type, as was the case for the analysis of $\mathrm{HO}$ effects performed here.

In the following, we will start by reporting the LRP-R findings. According to the RT partitioning performed in the present experiments, event repetitions leave activation traces that induce a tendency for cost-benefit effects in RTs. More specifically, as is indicated by the longterm LRP, amplitude repetitions leave motoric traces. If there are indeed motoric traces, they should affect the speed of motoric processing. Sequential patterns produced by such residual traces should mirror the cost-benefit pattern, with opposite slopes for the repetition and the alternation branches, together with an overall benefit for repetitions (FO repetition effect). Indeed, for continued repetitions, the LRP-R interval decreased with the length of such runs-that is, with the amount of relative trace accumulation. Interestingly, neither in Experiment 1 nor in Experiment 3 was there a significant $\mathrm{HO}$ effect in LRP-R intervals for sequences terminating with an FO alternation. This holds true even for Experiment 1, where such effects might have been expected in the first place because of the pure cost-benefit pattern in the RTs of this experiment. Therefore, it appears safe to say that on a motoric level, there are no costs when the traces do not correspond to the requirements of stimulus processing, despite clear benefits when they do correspond. A possible explanation for the lack of trace-dependent costs within the alternation branch might be that motoric traces accumulate only as long as the same event is repeated, but as soon as an alternative event occurs, the motoric trace is extinguished; a similar suggestion has been made by Pashler and Baylis (1991).

The results for the S-LRP interval suggest that most of the cost-benefit pattern with opposite slopes for the repetition and the alternation branches is located in premotoric processing stages. In terms of the trace account, this means that a premotoric mechanism is beneficial to the continued usage of the same trace and that, in contrast to motoric traces, the premotoric trace is not deleted when the alternative event occurs (for a review, see Treisman \& Williams, 1984). The finding of opposite slopes for the repetition and the alternation branches was clear in the first experiment, which used spatial compatible stimulus-to-response arrangements.
In Experiment 3, there was a positive slope in the S-LRP interval for the repetition branch, but no clear premotoric effect was found in the alternation branch. The absence of sequential effects in the alternation branch can be explained in two ways. Because Experiment 3 used a less compatible stimulus-response mapping than Experiment 1, it is possible that, here, the mechanisms that produce cost-only-seen in a rather pure form in Experiment 2-are present also at long RSIs. On the assumption that the cost-benefit pattern is also present in this experiment and that both mechanisms are located in premotoric stages, the positive slopes produced for the repetition branch by both mechanisms would add up. For the alternation branch, however, the mechanisms would produce opposite slopes that would cancel each other. Alternatively, it cannot be excluded that under the conditions of Experiment 3, neither the cost-only mechanism nor the cost-benefit mechanism was of sufficient strength to influence the observations. All that possibly remains at the premotoric level is a trace mechanism that shows the same one-sided characteristics as the motoric traces.

Taken together, the present results indicate that the cost-benefit effects as a function of the preceding stimulus sequence observed in performance have several sources. One mechanism or class of mechanisms appears to be the accumulation of traces from previous processing cycles that decay as a function of time. These traces affect the processing of the current stimulus at several levels. At motoric processing stages, existing traces corresponding to current processing requirements are beneficial, but noncorresponding traces evoke no costs. In contrast, at premotoric stages, corresponding and noncorresponding traces yield both benefits and costs, respectively. The contribution of premotoric stages to the performance effect appears to be more important than that of motoric stages.

A more general framework for the present findings may be provided by the idea of Kahneman and Tversky (1982) that there are different variants of expectancy. On the one hand, they suggest a more passive form of expectancy, which is said to be rather automatic and effortless, resembling priming. It yields a benefit if confirmed but no costs if disconfirmed. On the other hand, a more active variant of expectancy is considered to occupy consciousness and to draw on the limited capacity of attention. If subjects actively prepare for a specific event, its arrival will lead to benefits in performance. In contrast to passive expectancies, active expectancy will produce costs for unanticipated events. Both expectations are considered to work independently of each other and might have simultaneous effects on performance. The present findings of trace-dependent motoric benefits for repetitions might be seen as corresponding to Kahneman and Tversky's passive variant of expectancy. In contrast, in premotoric processing stages, sequencedependent benefits are balanced by corresponding costs. 
Therefore, in these stages, a more active variant of expectancy appears to be working, possibly in combination with passive expectancies. Future research should address the relative contributions of active and passive expectancies to premotoric processing and should specify their actions in perceptual or more central processing stages.

\section{REFERENCES}

Bertelson, P. (1963). S-R relationships and reaction times to new versus repeated signals in a serial task. Journal of Experimental Psychology, 65, 478-484.

Bertelson, P. (1965). Serial choice reaction time as a function of response versus signal-and-response repetition. Nature, 206, 217-218.

Bertelson, P., \& Renkin, A. (1966). Reaction times to new versus repeated signals in a serial task as a function of response-signal time interval. Acta Psychologica, 25, 132-136.

Biederman, I., \& Stacy, E. (1974). Stimulus probability and stimulus set size in memory scanning. Journal of Experimental Psychology, 102, 1100-1107.

Coles, M. G. H. (1989). Modern mind-brain reading: Psychophysiology, physiology, and cognition. Psychophysiology, 26, 251-269.

De Jong, R., Liang, C.-C., \& Lauber, E. (1994). Conditional and unconditional automaticity: A dual-process model of effects of spatial stimulus-response correspondence. Journal of Experimental Psychology: Human Perception \& Performance, 20, 731-750.

De Jong, R., Wierda, M., Mulder, G., \& Mulder, L. J. M. (1988). Use of partial stimulus information in response processing. Journal of Experimental Psychology: Human Perception \& Performance, 14, 682-692.

Duncan-Johnson, C. C., Roth, W. T., \& Kopell, B. S. (1984). Effects of stimulus sequence in $\mathrm{P} 300$ and reaction time in schizophrenics: A preliminary report. In R. Karrer, J. Cohne, \& P. Tveting (Eds.), Brain and information: Event-related potentials (Annals of the New York Academy of Sciences, Vol. 425, pp. 570-577). New York: New York Academy of Sciences.

ElLs, J. E., \& GotTs, G. H. (1977). Serial reaction time as a function of the nature of repeated events. Journal of Experimental Psychology: Human Perception \& Performance, 3, 234-242.

ENTUS, A., \& BindRA, D. (1970). Common features of the "repetition" and "same-different" effects in reaction time experiments. Perception \& Psychophysics, 7, 143-148.

Ford, J. M., Duncan-Johnson, C. C., Pfefferbaum, A., \& Kopell, B. S. (1982). Expectancy for events in old age: Stimulus sequence effects on P300 and reaction time. Journal of Gerontology, 37, 696-704.

HaLe, D. J. (1967). Sequential effects in a two-choice serial reaction task. Quarterly Journal of Experimental Psychology, 19, 133-141.

Hommel, B. (1994). Spontaneous decay of response-code activation. Psychological Research, 56, 261-268.

Jentzsch, I., \& Sommer, W. (2001). Sequence-sensitive subcomponents of P300: Topographical analyses and dipole source localization. Psychophysiology, 38, 607-621.

Kahneman, D., \& TVERSKY, A. (1982). Variants of uncertainty. In D. Kahneman, P. Slovic, \& A. Tversky (Eds.), Judgment under uncertainty: Heuristics and biases (pp. 509-520). New York: Cambridge University Press.

Kirby, N. H. (1972). Sequential effects in serial reaction time. Journal of Experimental Psychology, 96, 32-36.

KIRBY, N. H. (1976). Sequential effects in two-choice reaction time: Automatic facilitation or subjective expectancy? Journal of Experimental Psychology: Human Perception \& Performance, 2, 567-577.

KIRBy, N. H. (1980). Sequential effects in choice reaction time. In A. T. Welford (Ed.), Reaction times (pp. 129-172). London: Academic Press.

Kornblum, S. (1969). Sequential determinants of information processing in serial and discrete choice reaction time. Psychological Review, 76, 113-131.
Kornblum, S. (1975). An invariance in choice reaction time with varying numbers of alternatives and constant probability. In P. M. A. Rabbitt \& S. Dornic (Eds.), Attention and performance V (pp. 366-382). New York: Academic Press.

LAMING, D. R. J. (1968). Information theory of choice reaction times. London: Academic Press.

LAMING, D. R. J. (1969). Subjective probability in choice-reaction experiments. Journal of Mathematical Psychology, 6, 81-120.

Leuthold, H., Sommer, W., \& Ulrich, R. (1996). Partial advance information and response preparation: Inferences from the lateralized readiness potential. Journal of Experimental Psychology: General, 125, 307-323.

Melis, A., Soetens, E., \& van der Molen, M. W. (2002). Processspecific slowing with advancing age: Evidence derived from the analysis of sequential effects. Brain \& Cognition, 49, 420-435.

Miller, J., Patterson, T., \& Ulrich, R. (1998). Jackknife-based method for measuring LRP onset latency differences. Psychophysiology, 35, 99-115.

OLDFIELD, R. C. (1971). The assessment and analysis of handedness: The Edinburgh inventory. Neuropsychologia, 9, 97-113.

Osman, A., \& Moore, C. M. (1993). The locus of dual-task interference: Psychological refractory effects on movement-related brain potentials. Journal of Experimental Psychology: Human Perception \& Performance, 19, 1292-1312.

Pashler, H., \& BAYlis, G. C. (1991). Procedural learning: 2. Intertrial repetition effects in speeded choice tasks. Journal of Experimental Psychology: Learning, Memory, \& Cognition, 17, 33-48.

RaввітT, P. M. A. (1968). Repetition effects and signal classification strategies in serial choice-response tasks. Quarterly Journal of Experimental Psychology, 20, 232-240.

RAвbitT, P. M. A. (1980). The effects of R-S interval duration on serial choice reaction time: Preparation time or response monitoring time? Ergonomics, 23, 65-77.

Remington, R. J. (1969). Analysis of sequential effects in choice reaction times. Journal of Experimental Psychology, 82, 250-256.

Sмiтн, M. C. (1968). Repetition effect and short-term memory. Journal of Experimental Psychology, 77, 435-439.

SoETENS, E. (1998). Localizing sequential effects in serial choice reaction time with the information reduction procedure. Journal of Experimental Psychology: Human Perception \& Performance, 24, 547 568.

Soetens, E., Boer, L. C., \& Hueting, J. E. (1985). Expectancy or automatic facilitation? Separating sequential effects in two-choice reaction time. Journal of Experimental Psychology: Human Perception \& Performance, 11, 598-616.

Soetens, E., Deboeck, M., \& Hueting, J. (1984). Automatic aftereffects in two-choice reaction time: A mathematical representation of some concepts. Journal of Experimental Psychology: Human Perception \& Performance, 10, 581-598.

Sommer, W., Leuthold, H., \& Soetens, E. (1999). Covert signs of expectancy in serial reaction time tasks revealed by event-related potentials. Perception \& Psychophysics, 61, 342-353.

STERnberg, S. (1969). The discovery of processing stages: Extensions of Donders' method. In W. G. Koster (Ed.), Attention and performance II (pp. 276-315). Amsterdam: North-Holland.

Treisman, M., \& Williams, T. C. (1984). A theory of criterion setting with an application to sequential dependencies. Psychological Review, 91, 68-111.

Ulrich, R., \& Miller, J. (2001). Using the jackknife-based scoring method for measuring LRP onset effects in factorial designs. Psychophysiology, 38, 816-827.

Vervaeck, K. R., \& Boer, L. C. (1980). Sequential effects in twochoice reaction time: Subjective expectancy and automatic aftereffects at short response-stimulus intervals. Acta Psychologica, 44, 175-190.

WeLford, A. T. (1967). Single channel operation in the brain. Acta Psychologica, 27, 5-22.

Welford, A. T. (1976). Skilled performance: Perceptual and motor skills. Glenview, IL: Scott Foresman. 


\section{NOTES}

1. In this article, we will use the term cost-only pattern in order to be consistent, even though it is actually not possible to determine whether the HO effects in short RSI situations are cost- or benefit-only, because there is no defined baseline condition.

2. The linear trends are convenient measures to quantify HO sequential patterns without assuming linearity of the underlying processes.

3. As is suggested by Figures 1, 5, and 7, the speed of the preceding reaction is always correlated to the speed of the actual reaction. Fast preceding reactions produce faster current reactions. So, one may wonder whether the reported effects are due not to the speed of the preceded reaction, but merely to that of the current reaction. We tested this in all three experiments and did not find that the reported effects depended on current speed.

(Manuscript received August 15, 2000; revision accepted for publication January 31, 2002.) 